\title{
Handlungsoptionen und Grenzen kommunaler Selbstverwaltung: eine Fallstudie
}

von Joachim Jens Hesse

\section{Einleitung}

Die Überprüfung des Regierungs- und Verwaltungshandelns stellt eine kontinuierliche Aufgabe aller gebietskörperschaftlichen Ebenen dar. Zwar finden sich gelegentlich Hinweise darauf, dass durch den ubiquitären Einsatz des Reformbegriffs von einer gewissen „Übersättigung“ des Publikums wie der Fachöffentlichkeit gesprochen werden kann, doch gilt dies meist nur für die damit verbundene Reformrhetorik (samt ihrer „modischen“ Ausprägungen), nicht aber für die materiell zur Diskussion stehenden Fragen. So sollten die vergangenen beiden Jahrzehnte deutlich gemacht haben, dass eine kontinuierliche Modernisierung der Regierungsorganisation und eine darauf gerichtete Verwaltungspolitik zu den selbstverständlichen Verpflichtungen politisch-administrativer Funktionsträger gehören und den die Staats- und Verwaltungswissenschaften konstituierenden Disziplinen (also den Rechts-, Wirtschafts- und Sozialwissenschaften) entsprechenden Forschungsertrag abverlangen. Haushalterische Probleme, die demographische Entwicklung, das sich verändernde Verhältnis zwischen öffentlichem und privatem Sektor, die wachsende Transnationalisierung des öffentlichen Handelns und schließlich ein sich veränderndes Verständnis von Staatlichkeit, kommunaler Selbstverwaltung und hierauf bezogener Politiken machen eine dauerhafte Beschäftigung mit Fragen des Regierungs- und Verwaltungshandelns unausweichlich. Dabei treten zur Bewältigung massiv veränderter Rahmen- und Ausgangsbedingungen Verunsicherungen darüber, was eine zeitgemäße politisch-administrative Steuerung ausmacht, welcher Instrumente sie sich bedienen könnte und wie das Verhältnis zwischen gesellschaftlicher Selbststeuerung, zeitgemäßer Selbstverwaltung und öffentlicher Gewährleistung gestaltet sein sollte. Die damit verbundenen Umorientierungen sind bei Anbietern wie Nachfragern öffentlicher Leistungen von zahlreichen Anpassungsprozessen begleitet, erlauben, ja erzwingen aber auch innovatives Handeln, das allzu tradierten Vorgehens- und Interaktionsweisen zukunftsfähige Strukturen entgegenzusetzen sucht. 
Die Potentiale hierfür zu identifizieren und Handlungsoptionen aufzuzeigen, ist Aufgabe dieses Beitrags, wobei nach bereits vorliegenden eher gesamthaft ausgerichteten Analysen jetzt am Beispiel eines Flächenlandes, Niedersachsen, die Kommunalebene im Vordergrund steht. Sie bleibt im Rahmen tradierter Untersuchungen zum öffentlichen Handeln eher unterthematisiert, zum Schaden des wissenschaftlichen wie des politisch-,,praktischen“" Erkenntnisprozesses. ${ }^{1}$

\section{Der Fall Niedersachsen}

In den nachfolgenden Ausführungen summiert der Autor seine in mehrstufigen Untersuchungen vorgelegten Empfehlungen zur Fortentwicklung der niedersächsischen Kommunalstruktur. Er wählt hierzu die Form von Leitsätzen, an die sich wiederum komprimierte Textteile anschließen. Dies erlaubt, den komplexen empirisch-analytischen Untersuchungsansatz und die sich damit verbindenden methodischen Vorkehrungen für die Auswertung „kontrolliert“ zu halten und ermöglicht zugleich eine zusammenfassende Würdigung der vorgetragenen Empfehlungen. Aussagen zu einzelnen Gebietskörperschaften stellen zunächst die Ausnahme dar, finden sich aber in den abschließenden Textpassagen, samt kartographischer Darstellungen.

Die sich über einen mehrjährigen Zeitraum erstreckenden Untersuchungen stehen im Kontext zahlreicher Versuche, die Ausgangsbedingungen für das öffent-

1 Für den nachfolgenden Bericht wird auf gesonderte Belege verzichtet; umfassende Literatur- und Materialnachweise finden sich in den diesen Ausführungen zugrunde liegenden Publikationen: Hesse, J.J.: Kommunalstrukturen in Niedersachsen (das sog. "Grund- oder Hesse-Gutachten"), Berlin/Hannover, 2010; ders.: Kommunalstrukuren in Niedersachsen, Erste Fortschreibung, Berlin/Hannover, 2011; ders.: Kommunalstrukturen in Niedersachsen, Zweite Fortschreibung, Berlin/Hannover, 2012; ders.: Kommunalstrukturen in Niedersachsen: eine teilregionale Untersuchung für den Raum Wolfsburg-Gifhorn-Helmstedt, Berlin/Wolfsburg, 2011; ders.: Kommunalstrukturen in Niedersachsen: eine teilregionale Untersuchung für den Raum Südniedersachsen, Berlin/Göttingen, 2011; ders.: Kommunalstrukturen in Niedersachsen: eine teilregionale Untersuchung für den Raum Nordostniedersachsen, Berlin/Lüneburg, 2011; ders.: Evaluation der Arbeit und Wirkungsweise der Niedersächsischen Regierungsvertretungen (2005-2008), Berlin/Hannover, 2008; ders.: Förderung der interkommunalen Zusammenarbeit in Niedersachsen, Berlin/Hannover, 2006; ders.: Verwaltungsmodernisierung im Bereich der Raumordnung und Landesentwicklung, Berlin/Hannover, 2006; ders.: Staatliche Repräsentanz in den Regionen. Funktion, Aufgaben und Organisation von „Regierungsbeauftragten“, Berlin/Oldenburg, 2004. Eine Reihe dieser Arbeiten bildet zudem die Grundlage für nachfolgende Buchpublikationen im Rahmen der bei Nomos erscheinenden Schriftenreihe „Staatsreform in Deutschland und Europa: Der öffentliche Sektor im nationalen und internationalen Vergleich“; dies gilt u.a. für Hesse, J.J./Götz, A.: Kooperation statt Fusion? Interkommunale Zusammenarbeit in den Flächenländern, Bd. 1, 2006; Hesse, J.J.: Raumordnung und Landesentwicklung: Reformoptionen für ein tradiertes Politikfeld, Bd. 3, 2006; ders.: Handlungs- und zukunftsfähige Kommunalstrukturen: der Fall Niedersachsen, Bd. 15, 2011; ders.: Der Staat in der Fläche. Landesverwaltung ohne Mittelinstanz?, Bd. 14, 2011. 
liche Handeln in diesem zweitgrößten deutschen Flächenland zu verbessern, sie einem „Modernisierungsansatz“ zu unterlegen, der den zwischenzeitlich erheblich veränderten Rahmenbedingungen (ökonomisch, politisch, soziokulturell) folgt. Unter den diesbezüglichen wissenschaftlichen Bemühungen ragen im Zeitablauf zwei heraus: Zunächst das Gutachten der sogenannten WeberKommission, die ihren Abschlussbericht im März 1969 vorlegte und damit eine wichtige Grundlage für die Leitbilder im Rahmen der letzten allgemeinen niedersächsischen Gebietsreform auf Gemeinde- und Landkreisebene schuf. Allerdings wurden von den zahlreichen Empfehlungen der Kommission nur Teile umgesetzt, behaupteten sich bereits zu diesem frühen Zeitpunkt jene Beharrungskräfte, die mit Blick auf den Erhalt der kommunalen Selbstverwaltung einen weitergehenden, die Heterogenität der niedersächsischen Kommunalstrukturen kritisch überprüfenden Reformansatz verhinderten. So kam es in Verfolgung der Empfehlungen der Kommission zwar zu einer beträchtlichen Verringerung der Gemeindezahl, unterschritt man aber die für die Kreise und die kreisfreien Städte des Landes diskutierten Mindestwerte zum Teil erheblich. Schließlich erfüllten von den verbleibenden 38 Landkreisen zum 31.12.1980 nur 15 das Kriterium einer Mindesteinwohnerzahl von 150.000, 12 Landkreise verfügten zu diesem Zeitpunkt über weniger als 100.000 Einwohner, der Landkreis LüchowDannenberg wies mit lediglich 48.800 Einwohnern die geringste Einwohnerzahl aus.

Mit Begin der 15. Wahlperiode leitete das Land Niedersachsen deshalb eine grundlegende Modernisierung seiner Verwaltungsstrukturen ein. Die Auflösung der Bezirksregierungen und der konsequente Übergang von einem dreistufigen $z u$ einem zweistufigen Verwaltungssystem standen dabei im Mittelpunkt. Als bemerkenswert an diesem Ansatz erwies sich die Stringenz der Reformbemühungen, die man „ergebnisorientiert und zeitnah“ umzusetzen suchte. Die genannten Ziele und nachfolgenden Maßnahmen lassen sich zu drei Handlungsfeldern zusammenfassen und zwei Reformphasen zuordnen; beides nahm auf politische Vorgaben zu Personaleinsparungen und zum Systemwechsel sowie auf eine vergleichsweise umfassende Aufgabenkritik Bezug, die zumindest in Teilbereichen auch zu einem tatsächlichen Aufgabenverzicht führte. Im Ergebnis konzentrierte sich die niedersächsische Verwaltungspolitik zwar auf den Übergang zur Zweistufigkeit, doch trat eine sektorale Konzentration hinzu, die sowohl die Kommunalisierung und Verlagerung von Aufgaben auf Dritte als auch die Schaffung und fachliche Bündelung von Einrichtungen vorsah. Schließlich kam es mit dem Abbau von Standards und Normen, dem Verzicht auf Wider- 
spruchsverfahren und einem ,rationalisierten“ Personal- und Ressourceneinsatz zu einer „Optimierung“ von Querschnittsbereichen.

Wurde so die staatliche Verwaltung Niedersachsens einer grundlegenden Überprüfung und schrittweisen Modernisierung unterzogen, wandte sich die Landesregierung schließlich auch der kommunalen Ebene zu, in der berechtigten Erwartung, dass hier nicht nur der Bedarf, sondern auch die Bereitschaft zu einer komplementären Überprüfung der dezentralen Verwaltungsstrukturen gegeben sei. Dieser Prozess wurde seit dem Jahr 2005 vom Internationalen Institut für Staats- und Europawissenschaften (ISE) in Berlin begleitet, wobei der Autor rückblickend auf eine Reihe von Untersuchungen verweisen kann, die sich Schlüsselfragen des Modernisierungsansatzes widmeten. Dies gilt zum einen für jenen Beitrag, der im Rahmen der ersten Stufe des niedersächsischen Reformprozesses nach der sich damit verbindenden Rolle und Funktion des Staates ,in der Fläche" fragte, setzte sich fort in umfassenden Untersuchungen zu den Ansätzen und Ergebnissen interkommunaler Zusammenarbeit, bezog eine Überprüfung der traditionellen Raumordnung und Landesplanung ein und mündete schließlich in eine Evaluation der Regierungsvertretungen, also jener Einrichtungen, die nach Abschaffung der Bezirksregierungen Aufgaben einer regionalen „Entwicklungsagentur“ wahrnehmen sollten. Die im Verlauf dieser Untersuchungen gewonnenen Erkenntnisse wurden - ergänzt um themenspezifisch ähnliche Arbeiten in inzwischen allen Flächenländern der Bundesrepublik - unter dem Titel „Was soll und kann Verwaltungsreform? Der Fall Niedersachsen“ im Jahr 2007 zusammengefasst.

Die wissenschaftliche Begleitung der hier im Zentrum stehenden Kommunalreform schloss sich an, wobei drei im Jahresrhythmus aufeinander folgende Untersuchungen vorgelegt wurden: zunächst der Bericht „Handlungs- und zukunftsfähige Kommunalstrukturen“" Mitte 2010, das sog. „Grund- oder auch HesseGutachten", dann dessen erste Fortschreibung Ende 2011 sowie die zum 31.12.2012 vorgelegte und hier vorgestellte zweite Fortschreibung, die als Abschlussbericht gilt. Darüber hinaus wurden für drei „Problemräume“ des Landes gesonderte Untersuchungen erstellt, die seitens der betroffenen Gebietskörperschaften in Auftrag gegeben wurden. Sie richteten sich, in dieser Reihenfolge, auf den Raum Wolfsburg-Gifhorn-Helmstedt, die Kommunalstrukturen in Nordostniedersachsen sowie schließlich auf die Entwicklungsprobleme Südniedersachsens .

Im der Zusammenfassung wird erkennbar, dass Niedersachsen - wie die anderen Flächenländer der Bundesrepublik - gut beraten wäre, einer konsequenten Über- 
prüfung seiner Kommunalstrukturen nicht länger auszuweichen. Die deutliche Veränderung nahezu aller Rahmenbedingungen, die massiven haushalterischen wie demographischen Verwerfungen, die in Teilen prekäre Entwicklung der kommunalen Selbstverwaltung (samt des sie tragenden Ehrenamtes) sowie das herannahende „Schlüsseljahr“ 2019 dürften eine reformorientierte Politik unausweichlich machen. Da zu dem benannten Jahr nicht nur die Strukturhilfen (Solidarpakt II) auslaufen, sondern auch der Länderfinanzausgleich erneuert werden muss und (nahezu) alle Gebietskörperschaften sich zur Umsetzung der „Schuldenbremse“ verpflichtet haben, erhöht sich der Anpassungsdruck; addiert man dem, dass es in diesem Zeitraum auch zu komplementären Umorientierungen der Förderverfahren der Europäischen Union, des Bundes und der Länder kommen wird, erschließt sich die Größe der Aufgabe. Insofern wird eine dem staatlichen Reformansatz komplementäre Überprüfung der niedersächsischen Kommunalebene dringlich, bietet es sich für alle Beteiligten an, diesbezügliche Diskussionen nicht abzuwehren, sondern sie aktiv (mit) zu gestalten.

\section{Unabweisbarer Handlungsbedarf}

Die im Vergleich sehr heterogenen Kommunalstrukturen Niedersachsens bedürfen eines umfassenderen Reformansatzes vor allem dann, wenn die gegebenen Organisationseinheiten und die erkennbare Leistungsfähigkeit der Akteure dies nahe legen - in eigener Verantwortung oder (bei Ausbleiben) extern induziert. Zur Beurteilung eines etwaigen Änderungsbedarfs erscheint es, auch im historischen Längsschnitt, zunächst wichtig, sich der sich mit der Reform des staatlichen Bereichs verbindenden Konsequenzen für die Kommunalebene zu erinnern, nicht zuletzt mit Blick auf übertragene Aufgaben und den mit dem Rückzug des Staates aus der Fläche verbundenen Koordinationsbedarf. Zudem wurde deutlich, dass die von der Kommunalebene selbst initiierten Reformansätze nicht ausreichten, die heterogenen Handlungskapazitäten, die beträchtlichen regionalen Disparitäten und die sich verschärfenden sozioökonomischen wie demographischen Rahmenbedingungen zu bewältigen. Die Landesregierung sah deshalb im Rahmen der zweiten Stufe der Verwaltungsmodernisierung zunächst eine verstärkte Förderung interkommunaler Zusammenarbeit vor, erst später ergänzt um die immer wieder diskutierten, aber erwartungsgemäß hochgradig strittigen Struktureingriffe, die von gleichfalls freiwilligen Lösungen über finanzielle Anreize bis hin zur Inaussichtstellung gesetzgeberischer Akte reichten - vor allem für den Fall, dass sich die Ergebnisse der Kooperationsstrategie im Ergebnis als (zu) begrenzt erweisen sollten. 
Ausgehend von einem im Ländervergleich als bestenfalls durchschnittlich zu charakterisierenden Niveau der interkommunalen Zusammenarbeit stellte sich anfangs die Frage, in welchen Bereichen und in welcher Form sich deren Intensivierung anbot und Erfolg versprechend erschien. Diesbezügliche Expertengespräche und eine flächendeckende Befragung zur niedersächsischen Ausgangssituation führten zu der Erkenntnis, dass sich neben einem Nachholbedarf im kreisangehörigen Raum hierfür vor allem eine verbesserte (vertikale) Kooperation zwischen der Kreisstufe und den Gemeinden anbot. Hinzu traten die Überwindung eines erkennbaren Rückstandes in Süd- und Ostniedersachsen sowie der Ausbau von Stadt-Umland-Bezügen. Instrumentell wurde die Konzentration auf einfache vertragliche Formen der Mitverwaltung empfohlen, um auf diesem Weg die Schaffung neuer Einheiten mit eigenständigen Organisationsinteressen zu vermeiden. Materiell ging es dagegen um die Zusammenarbeit in der allgemeinen Verwaltung (etwa im Personal- und Beschaffungswesen), daneben auf der Kreisstufe um die Kooperation in Sonderordnungsbereichen (Veterinär- und Lebensmittelaufsicht als Beispiel) sowie insgesamt um den Ausbau entwicklungspolitischer Zusammenhänge (etwa in Form interkommunaler Gewerbegebiete), ohne dafür freilich neue intermediäre Strukturen auszubilden.

Da der Handlungsansatz einer Kooperationsförderung grundsätzlich auf dem Prinzip der Freiwilligkeit beruht, konnte das Land zunächst nur flankierend tätig werden, zumal sich direkte Eingriffe zur Herstellung bestimmter Arbeitszusammenhänge verboten. Stattdessen ging es um die Erhöhung der kontextabhängigen Motivation zur Zusammenarbeit. Diese ist vor allem dann gegeben, wenn der in Aussicht gestellte Gewinn (Personaleinsparungen, verbesserte Verwaltungsqualität und künftige Steuermehreinnahmen als Beispiele) und die zu erwartenden politisch-administrativen Nachteile einer Nicht-Kooperation (etwa in Form einer sonst befürchteten Gebietsreform) zusammengenommen die Summe der unmittelbaren Kosten (Aufwendungen für Planung und Anbahnung der Zusammenarbeit, Kontrollaufwand gegenüber Gemeinschaftseinrichtungen usw.), des Autonomieverlusts und der politisch-administrativen Nachteile der Kooperation (etwa durch eine dadurch befürchtete Vorwegnahme von Gebietsreformen) übersteigen. Das Land kann hierbei vor allem verstärkend wirken, indem es einen höheren materiellen Gewinn in Aussicht stellt, durch rechtliche Rahmenbedingungen fortgesetzte Nicht-Kooperation diskriminiert, die unmittelbaren Kooperationskosten und rechtlichen Hürden absenkt sowie politisch-administrative Nachteile durch die Definition klarer Rahmenbedingungen und Konsequenzen einer vom Land geförderten Gemeinschaftsarbeit begrenzt. Seitens der kommu- 
nalen Gebietskörperschaften geht es darum, einen angemessenen Eigenbeitrag zu erbringen. Hierzu zählen neben der grundsätzlichen Offenheit für kooperatives Handeln die Bereitschaft zur Wahrnehmung einer Initiatorenrolle, die Ausgestaltung einer inhaltlich und im Verfahren realistischen Kooperationsagenda, die Schaffung möglichst wenig kontrollintensiver und nicht zur Verselbständigung neigender Organisationslösungen, die Vereinbarung einer wechselseitig akzeptierten und auch funktional längerfristig tragenden Kosten- und Nutzenverteilung sowie die Einrichtung einer ergebnisorientierten Prozesssteuerung.

Im Rahmen der benannten Untersuchung wurde dem Land vorgeschlagen, zunächst ein breites Spektrum unterschiedlich aufwendiger und eingriffsintensiver Maßnahmen zu prüfen, das von der Information und Moderation gegenüber dezentralen Entscheidungsträgern über Managementhilfen und personelle Unterstützung bis hin zur Berücksichtigung im kommunalen Finanzausgleich und dem Setzen negativer Referenzpunkte in Form ,angedrohter" Strukturreformen reichte. In der Umsetzung wurde für ein Vorgehen in mehreren Stufen votiert, das zunächst eine verbesserte Selbstregelung der Kommunen (unterstützt durch Beratungsleistungen, einen dezentralen Vollzug der Kommunalaufsicht und kooperationsbezogene Deregulierungen im Landesrecht) und eine Anreizsteuerung durch das Land vorsah (Managementhilfen, Kooperationsfonds für projektspezifische Förderung, flexible größenabhängige Delegation von staatlichen Aufgaben, kooperationsfreundliche Ausnahmetatbestände im Raumordnungsrecht).

Entsprechende Maßnahmen wurden im Land dann auch diskutiert, ohne dass dies freilich zu einem hinreichenden Konsens geführt hätte; von einer signifikanten Anhebung des Kooperationsniveaus konnte nicht gesprochen werden. Damit wurden allerdings jene Vorbehalte bestätigt, die der Autor bereits im Rahmen früherer Untersuchungen formulierte. Sie bezogen sich auf die zu veranschlagenden Kooperationskosten, den Zeitverzug durch eine jeweils einzelthematische Realisierung, die damit verbundenen politisch-administrativen Hürden und Widerstände, den Verbleib eines von den Kommunen kaum zur Disposition gestellten Autonomiebesatzes (im Bereich von Kernaufgaben und Hoheiten) sowie die Gefahr einer kooperativen Sättigung aufgrund des infolge fortschreitender Gemeinschaftsarbeit befürchteten Autonomieverlusts. Deshalb empfahl er, die (freiwillige) Selbstregelung und (flankierende) Anreizsteuerung durch eine Strukturförderung zu ergänzen, die eine finanzielle Unterstützung und selbsttätig gestaltete Fusionen beinhalten sollte. Nach einer Start- und Förderphase müsste dieser Ansatz evaluiert und auf dieser Basis dann entschieden werden, inwieweit 
das Land in Form von Strukturvorgaben und Mindestgrößen in den kommenden Jahren einen Handlungskorridor vorgibt, der durch die Ankündigung gesetzlicher Maßnahmen begrenzt wird und damit die Handlungsbereitschaft der Kommunen in kooperativer wie organisatorischer Hinsicht verstärkt. Diese Vorschläge zogen erwartungsgemäß Kritik auf sich, erwiesen sich aber vor dem Hintergrund der schwierigen Finanzlage, der ungleichgewichtigen Kapazitäten und der kooperativ nur sehr begrenzt zu bewältigenden Rahmenbedingungen als gleichsam ,alternativlos".

Hinzu tritt, dass auch Reformerfordernisse in Querschnittsbereichen, insbesondere die bereits angesprochene Stärkung der horizontalen Koordinationsfähigkeit und eine Weiterentwicklung der Raumordnung und Landesentwicklung, mittelund langfristig organisationsstrukturelle Anpassungen erforderlich machen; nur auf diese Weise lassen sich ausreichende Einzugs- und Wirkungsbereiche herstellen, die einen kompetenten und vor allem effektiven Vollzug komplexer und längerfristig angelegter Aufgaben gestatten (die Regionalentwicklung als Beispiel). Blickt man in diesem Zusammenhang auf die Frage einer möglichen Anpassung der Gebietsorganisation auch auf der kommunalen Kreisstufe, steht dies notwendigerweise unter dem Vorbehalt eines verschärften Begründungszwangs. Demnach wären sowohl bei territorialen als auch bei bloßen organisatorischen Anpassungen (etwa durch die Bildung von obligatorischen Planungsverbänden) die Ausgewogenheit und Funktionsfähigkeit gegebener Strukturen zu berücksichtigen, nachgewiesene Leistungsdefizite und erkennbare Disparitäten abzubauen und eine auch im Vergleich effektive Bewältigung der sozioökonomischen und demographischen Probleme als Kriterien für eine prospektiv begründete Strukturentwicklung zugrunde zu legen. In der Konsequenz dürften sich verstärkte Anforderungen an freiwillige interkommunale Zusammenarbeit im Ordnungs- und Entwicklungsbereich kaum als strittig erweisen. Desiderat bliebe dabei eine Harmonisierung von Einzugsbereichen im Rahmen mittelfristig anzustrebender Mindestgrößen von 150.000 bis 200.000 Einwohnern (bei stabiler demographischer Entwicklung). So verweist auch die Modernisierung in Querschnittsbereichen auf die Notwendigkeit (die Raumordnung hier erneut als Beispiel benannt), den mit den ersten beiden Stufen der niedersächsischen Verwaltungsreform eingeschlagenen Kurs fortzuführen und über (zunächst) kooperative Strategien auf den kommunalen Bereich auszuweiten. Bei deren Ausbleiben wäre der Gesetzgeber gefordert.

Im Ergebnis bot es sich mithin an, den Reformansatz zunächst unter das Freiwilligkeitsgebot zu stellen und über einen zwischen dem Land und den kommunalen 
Spitzenverbänden geschlossenen „Zukunftsvertrag“ eine Reihe von Anreizen vorzusehen, sich auf Veränderungen (bis hin zu Fusionen) einzulassen. Heute freilich ist festzuhalten, dass trotz zahlreicher Diskussionen diese Angebote des Landes nur unzureichend wahrgenommen wurden und auch die kommunalen Spitzenverbände entsprechende Bemühungen eher schwächten als sie aktiv zu befördern. Zudem wurde im politischen Raum erkennbar, dass das von Beobachtern immer wieder angeregte überparteiliche Handeln letztlich nur in Teilräumen oder punktuell praktiziert wurde. Der Landtagswahlkampf schließlich (Ende 2012/Anfang 2013) verfestigte die jeweiligen Grundpositionen, die materiell zwar kaum mehr trugen, in der Konfrontation mit dem politischen Gegner aber noch zu taugen schienen. Derzeit findet sich keinerlei Hinweis auf ein parteiübergreifendes Denken (oder gar Handeln), eine nicht nur angesichts der fragilen Ein-Stimmen-Mehrheit der regierenden rot-grünen Koalition problematische Ausgangssituation.

Die in den vergangenen drei Jahren ermöglichte Beobachtung der für die Kommunalstrukturen Niedersachsens wichtigsten Akteure hinterlässt zudem ein ambivalentes Bild. So findet sich zwar eine schmale Gruppe sachkundiger Reformpromotoren, die auf laufende wie absehbare Veränderungsprozesse hinweist und sich um Konsens zu einzelnen Reformschritten bemüht, doch steht dem eine breite Mehrheit gegenüber, die entweder eine abwartende Haltung einnimmt (bzw. nur das Land in der Gestaltungsrolle sieht) oder aber reformbezogene Diskussionen zu verhindern, wenigstens aber zu blockieren sucht. Dies ist nach den Erfahrungen in fast allen Flächenländern der Bundesrepublik ein zwar nicht unbekanntes, gleichwohl aber bedauerliches Verhalten, da es ein nicht eben zukunftsorientiertes Denken dokumentiert. In der Konsequenz findet sich der von Sachkundigen beklagte „Flickenteppich“von Reformaktivitäten, der eher der jeweiligen finanziellen Entlastung (Entschuldungshilfen im Rahmen des Zukunftsvertrags) als einer konsequenten und kohärenten Reformpolitik geschuldet sein dürfte. Darüber hinaus kommt es zwar zu freiwillige Gemeindefusionen und (wenigen) dementsprechenden Diskussionen auch auf der Kreisebene, doch bezieht sich das meist auf die Problemräume im Osten und Süden des Landes. Im Ergebnis sucht man jedweden „Eingriff“ in den kommunalen Besitzstand abzuwehren, ohne die Entwicklungschancen zu sehen (oder sie nutzen zu wollen), die sich mit einem kooperativeren Handeln verbinden. Das erkennbare Warten auf Vorgaben, mithin eine „Steuerung“ des Landes, könnte sich durchaus rächen, insbesondere dann, wenn die angesprochenen Disparitäten letztlich doch ein „Leitbild“ erforderten, nach dem etwa Kreise unterhalb einer Einwohnerzahl 
von 150.000, kreisfreie Städte unterhalb der 100.000 Einwohner-Grenze und schließlich Gemeinden mit weniger als 10.000 Einwohnern in besonderer Weise in ihrem Bestand gefährdet wären, der Gesetzgeber die Beweislast mithin umkehrte.

\section{Vom Sinn und Unsinn der Leitbilder: Plädoyer für Entscheidungen im Einzelfall}

Wie bereits in dem „Grundgutachten“ umfassend dokumentiert, spricht sich der Autor mit Blick auf etwaige Gebietsreformen gleichwohl für eine Würdigung des jeweiligen Einzelfalls aus. Dies ergibt sich aus der bereits mehrfach angesprochenen Heterogenität des Landes, samt seiner ausgeprägten Disparitäten und damit unterschiedlichen Entwicklungspotentiale. So bleiben der Süden und der Osten des Landes auf absehbare Zeit ,gefährdet“", sei es aufgrund der Bevölkerungsentwicklung oder aber der jeweiligen Haushaltssituation, während das Zentrum und vor allem der Westen des Landes weniger dringenden Handlungsbedarf erkennen lassen. Die „Küstenregion“ steht hingegen in einer Übergangssituation, die sich mit erfolgreichen wirtschafts- und infrastrukturpolitischen Maßnahmen verbindet und bei einem komplementären Zusammenwirken der kommunalen Akteure beträchtliche Handlungspotentiale (etwa über den JadeWeserPort) birgt. Diese zwar zu sehen, sie jetzt aber erneut eher „kleinteilig“ anzulegen, könnte sich insofern als verhängnisvoll erweisen, als davon nicht nur deutsche, sondern vor allem auch niederländische und andere europäische Wettbewerber profitieren dürften.

Dass im Übrigen ,größer nicht unbedingt besser" sein muss, um eine triviale Argumentation im Rahmen der gegenwärtigen Diskussion aufzunehmen, ist allen Informierten bekannt. Allerdings sollte gleichermaßen bekannt sein, dass sich sowohl unter- wie überdimensionierte Organisationsstrukturen („BetriebsgröBen“) nicht „auszahlen“, weder ökonomisch noch demokratiepolitisch. Economies of scale und economies of scope (also Größen- und Verbundvorteile) produktiv zu nutzen, müsste mithin im Interesse nicht nur der Landes-, sondern auch der kommunalen Akteure sein. Zu den kosten- wie leistungswirksamen Effekten einer etwaigen kommunalen Gebietsreform treten im Übrigen Entwicklungsprozesse, die sich in den vergangenen Jahren deutlich verstärkt haben und einer erweiterten Beachtung bedürfen: hierzu zählen eine gewisse (Re-) Zentralisierung der Siedlungsstruktur wie der Leistungserbringung (zugunsten der städtischen Räume), die Anforderungen einer immer großflächigeren Infrastrukturversorgung, aber auch laufende Umorientierungen bei der Gestaltung des 
Finanzausgleichs, etwa hin zu einer stärkeren Gewichtung von Wachstumsgegenüber Ausgleichszielen. Dass dies leistungsfähige gebietskörperschaftliche Akteure und eine noch intensivere Kooperation mit der örtlichen, regionalen und nationalen Wirtschaft erfordert, sollte unstrittig sein.

Schließlich gilt aber auch für die Diskussion von territorialen Größenordnungen und etwaigen „Leitbildern“, dass die kommunale Selbstverwaltung ein in jeder Hinsicht (aber nicht um jeden Preis) zu schützendes Gut darstellt; der Autor hat dies in zahlreichen Untersuchungen zu dokumentieren versucht und fallstudienartig für die meisten deutschen Flächenländer belegen können. Er ist derzeit auch Gutachter in Mecklenburg-Vorpommern, das nach langer strittiger Auseinandersetzung Großkreise formte, nicht aufgrund einer besonderen Präferenz für derartige Gebilde, sondern angesichts der dramatischen, die Selbstverwaltung des Landes existentiell gefährdenden Bevölkerungsverluste. In einer auf drei Jahre angelegten Begleituntersuchung werden hier, eine vom Landesverfassungsgericht geforderte Beobachtungspflicht einlösend, die Wirkungen der Kreisstrukturreform, insbesondere auf das Ehrenamt, verfolgt.

\section{Ein breitestmöglicher Indikatorensatz zur Beurteilung der Kommunal- struktur}

Um der Ausgangssituation in Niedersachsen umfassend gerecht zu werden, stellte der Autor schließlich (nach einer umfassenden Erörterung der veränderten Rahmenbedingungen) eine ungewöhnliche Zahl quantitativer wie qualitativer Maßstäbe, Kriterien und Indikatoren zur Beurteilung der Kommunalstruktur zusammen. Deren Anwendung ermöglicht es, angesichts der geschilderten Ausgangssituation und der erwartbaren Entwicklung aufzuzeigen, ob und wo im Land Stabilisierungs- und ggf. Handlungsbedarf bestehen. Hierzu wurde die bereits im „Grundgutachten“ angelegte Indikatorenanalyse kontinuierlich aktualisiert und in Teilen verfeinert. Gewählt wurden neben dem jeweiligen Bevölkerungsbesatz und der Raumkapazität die verwaltungsgeographische Kongruenz, die Entwicklungsfähigkeit sowie die sozioökonomische und fiskalische Ausgleichsfähigkeit der Gebietskörperschaften; Indikatoren zur ebenenübergreifenden Funktionalität und verwaltungspolitischen Stabilität sowie zur örtlichen, demokratischen und politischen Integrationsfähigkeit traten hinzu (vgl. Tab. 1).

Mit Hilfe dieses Indikatorenbündels, das wiederum auf einer breiten Begründung aufbaute und um zusätzliche vor Ort gewonnene Erkenntnisse ergänzt wurde, 
war es möglich, die kommunalen Gebietskörperschaften Niedersachsens einer umfassenden Beurteilung und Bewertung zu unterziehen. Dabei stand zunächst die Kreisebene im Vordergrund, ergänzt um entsprechende Aussagen für den gemeindlichen Bereich. Darüber hinaus wurden die Ausprägungen für die einzelnen kreisfreien Städte, die Landkreise und die Region Hannover jeweils in Tabellenform dargestellt, wobei der Vergleich die Herausbildung von drei Gruppen erlaubte: Kommunen mit hohem, mittlerem und geringem/nicht gegebenem Stabilisierungs- und/oder Handlungsbedarf. Diese Vorgehensweise, mit Teilen der Fachöffentlichkeit abgestimmt, gilt derzeit als „Referenz“ und wird auf weitere Flächenländer übertragen. Sie ist zudem Teil der im ISE Berlin aufgebauten und kontinuierlich erweiterten „Strukturberichterstattung für die deutschen Gebietskörperschaften".

Tabelle 1: Übersicht über die eingesetzten Indikatoren (Kreise)

\section{Bevölkerungsbesatz und Raumkapazität}

- Flächengröße der Landkreise (in $\mathrm{km}^{2}$ )

- Einwohnerzahl

\section{Verwaltungsgeographische Kongruenz}

- Arbeitsplatzeigenversorgung: Anteil derjenigen Personen, die in einer Kommune wohnhaft und sozialversicherungspflichtig beschäftigt sind, an allen sozialversicherungspflichtig Beschäftigten, die in der Kommune wohnen (in Prozent)

- Abdeckung von Arbeitsmarktregionen: Anteil der Einwohner einer Kommune an der Bevölkerung der örtlichen Arbeitsmarktregion (in Prozent)

- Abdeckung von Naturräumen: Zahl zumindest teilweise abgedeckter naturräumlicher Regionen und Zahl weiterer Kommunen in denselben Naturräumen

- Güte der Korrespondenz mit grenzüberschreitenden Bezügen anhand einer qualitativen Einschätzung

\section{Entwicklungsfähigkeit}

3.1 Sozioökonomische Entwicklungsfähigkeit

- Bruttoinlandsprodukt je Einwohner (in Euro)

- Arbeitslosenquote (bezogen auf alle zivilen Erwerbspersonen; in Prozent)

- Veränderung der Zahl der Beschäftigten im Jahr 2030 ggü. 2010 (in Prozent)

3.2 Demographische Entwicklungsfähigkeit

- Veränderung der Einwohnerzahl im Jahr 2030 ggü. dem Jahr 2011 (in Prozent)

- Veränderung des Bevölkerungsanteils der unter 18-Jährigen von 2011 bis 2030 (in Prozent)

- Veränderung des Bevölkerungsanteils der über 75-Jährigen von 2011 bis 2030 (in Prozent) 


\subsection{Haushalterische Entwicklungsfähigkeit}

- Deckungsquote im Jahresdurchschnitt 2008-2010 (Verhältnis der allgemeinen Deckungsmittel netto zu den Zuschussbedarfen der Einzelpläne 0-8 des Verwaltungshaushalts; in Prozent)

- Zinsquote im Jahresdurchschnitt 2008-2010 (Anteil der Zinsausgaben an den allgemeinen Deckungsmitteln brutto; in Prozent)

4. Sozioökonomische und fiskalische Ausgleichsfähigkeit

- Zentralörtliches Versorgungsniveau: Quote der Einwohner einer Kommune, die in einem Zentralen Ort wohnen, wozu hier die Ober- und Mittelzentren des Landes gerechnet werden, an deren gesamter Einwohnerschaft (in Prozent)

- SGB II-Quote: Anteil der hilfebedürftigen Personen nach dem SGB II (erwerbsfähige und nicht erwerbsfähige Hilfebedürftige) an der Bevölkerung bis unter 65 Jahren (in Prozent)

- Kommunale Steuereinnahmen pro Einwohner im Jahresdurchschnitt 2009-2011 (in Euro)

5. Ebenenübergreifende Funktionalität und verwaltungspolitische Stabilität

- Horizontales Gleichgewicht der Gemeindeverbände: Mittelwert der Anteile einer Kommune an der Einwohnerzahl, Flächengröße und den kommunalen Steuereinnahmen des Landes Niedersachsen (keine Bewertung einzelner Kommunen, sondern Bewertung der Kreisebene insgesamt; in Prozent)

6. Ortsnähe, Teilhabe und Identität

6.1 Teilhabe

- Zahl der Bewerber um ein Mandat in den Vertretungskörperschaften der Kreisstufe je Einwohner und Mandat bei den Kommunalwahlen in den Jahren 2006 und 2011

- Durchschnittliche Wahlbeteiligung bei den Kommunalwahlen in den Jahren 2006 und 2011 (in Prozent)

6.2 Ortsnähe

- Flächengröße der Landkreise (in $\mathrm{km}^{2}$ ) und damit verbundene Distanzen

6.3 Gegebene Identitäten im Zeitablauf

- Mittelwert der Flächenanteile einer Kommune: (1) im Territorium des 16. Jahrhunderts, (2) im Regierungs- oder Verwaltungsbezirk der Jahre 1946-1978, (3) heute (in Prozent)

\section{Wachsende Bekenntnis zur „Größerräumigkeit“", nicht nur in den Prob- lemregionen des Landes}

Die problematischen Entwicklungen im Süden und Osten Niedersachsens führten nach Vorlage des „Grundgutachtens“ zu der bemerkenswerten Entscheidung kommunaler Akteure, den Autor um eine gesonderte Untersuchung Nordostniedersachsens, des Raums Wolfsburg-Gifhorn-Helmstedt sowie Südniedersachsens 
zu bitten. Dem wurde mit den benannten Sondergutachten entsprochen, die eine umfassende regionale und in Teilen auch landesweite Diskussion auslösten. Wurde ursprünglich erhofft, hieraus gegebenenfalls einen „Nukleus“ für einen landesweiten Reformansatz bilden zu können, so scheiterte dies bald an der sehr ungleichen Ausgangssituation der benannten Räume. Gleichwohl wurden die erkennbaren Handlungsoptionen in ihrer normativen wie funktionalen „Streitigkeit" deutlich: etwa in der Frage, ob Nordostniedersachsen sich nicht doch sehr viel eher der Entwicklungsdynamik des Großraums/der Metropolregion Hamburgs anschließen sollte als interregionale Ausgleichs- und Solidarleistungen (etwa gegenüber Lüchow-Dannenberg und Uelzen) zu erbringen; stellte sich im Großraum Wolfsburg die Frage, ob man der starken Verflechtung im Raum eher durch eine Aufwertung des Oberzentrums Wolfsburg (etwa durch Eingemeindungen) oder aber durch eine den Verflechtungsprozessen folgende Kooperation und nachfolgende Fusion mit den Kreisen Helmstedt und Gifhorn (,Autoregion,,) gerecht werden könnte; und war schließlich mit Blick auf Südniedersachsen zu erörtern, ob der Randlagigkeit des Raums eher durch einen von Göttingen, Northeim und Osterode zu bildenden Großkreis, mithin eine gebündelte Interessenvertretung, zu begegnen sei oder ob Osterode in eine dem Westharz dienende Entwicklungsperspektive „,entlassen“ werden sollte.

In diesen Schlüsselentscheidungen, die von entsprechenden politischen Polarisierungen begleitet waren, wurden gleichsam exemplarisch die Vorteile wie Widersprüche gebietsreformerischer Optionen deutlich. Zur Bildung etwaiger „Großkreise" traten deshalb Strategien, eher die Kreisfreiheit (nicht nur Wolfsburgs, sondern auch Göttingens) zu befördern, galt es zudem, die Rechtsprechung der Landesverfassungsgerichte zu berücksichtigen und etwaige Fusionsprozesse an einer Reihe nachfolgender Kriterien zu messen. Schließlich suchte der Autor die sich vertiefenden Auseinandersetzungen auch noch durch den Ausweis kleinräumiger Verflechtungsbeziehungen zu versachlichen, zumal in einzelnen Fällen veränderte politische Mehrheiten weitergehende Begehrlichkeiten erkennen ließen. 


\section{Voraussetzungen der Reform}

\section{Eine diskussionswürdige Ausgangssituation: wachsende politische Polarisierung, fehlende funktionale Orientierung und unzureichende Kooperationsbereitschaft}

Sucht man die nicht nur in den benannten Problemregionen gemachten Erfahrungen der vergangenen Jahre zusammenzufassen, so wird erkennbar, dass funktionale Argumente, die sich angesichts unbestreitbarer Defizite vor allem auf die ökonomische und demographische Entwicklung von Einzelräumen richteten, eine eher geringe, institutionelle und personelle Erörterungen dagegen eine wachsende Bedeutung erfuhren. Das sollte in einem Flächenland, dessen politische Ausgeglichenheit aufgrund zweier fast gleichstarker Parteienblöcke ein wesentliches Strukturmerkmal ist, nicht verwundern, stellt allerdings eine Erschwernis dar. So blieb der Handlungsbedarf im Land zwar unbestritten, doch folgten dem, auch und gerade seitens der kommunalen Ebene, nur zögerliche Reaktionen. Dies gilt nicht nur für die schleppende Aufnahme des seitens der Landesregierung angebotenen Entschuldungsprogramms (und der sich damit verbindenden Entlastung der Kommunalhaushalte), sondern auch für die seit längerem geforderte (und zwischenzeitlich nachdrücklich geförderte) Ausweitung der interkommunalen Kooperation; es fanden sich nur wenige Pilotprojekte, die kaum eine nachhaltigere kommunale Gemeinschaftsarbeit nach sich zogen.

Insofern bietet es sich an, ggf. landesseitig die Selbstblockaden abzubauen und für die Kommunalreform ein Handlungskonzept zu entwickeln, das gesamthaft trägt, ohne die benannten Heterogenitäten/Disparitäten zu überspielen. Immer dann, wenn Freiwilligkeit betont und Anreizpolitiken für ein erweitertes Handeln zwar angeboten, aber nur unzureichend wahrgenommen werden, stellt sich für das Land (und das gilt für jedwede Landesregierung) die Aufgabe, den unabweisbaren Wandel zu gestalten. Dabei gilt es zum einen, das immer prekäre Gleichgewicht zwischen dem städtischen und dem ländlichen Raum in Niedersachsen zu erhalten, zugleich aber auch jene Wachstumspotentiale zu fördern, ohne die das Land im Wettbewerb der Flächenländer zurückfallen dürfte. Niedersachsen wird sich eingestehen müssen, dass nach der über die Abschaffung der Bezirksregierungen mutigen Entscheidung, eine nur noch zweistufige Verwaltung vorzusehen, diesem Reformschritt komplementäre Anpassungen der kommunalen Ebene bislang ausstehen. Sie auch in der jetzt beginnenden neuen Legislaturperiode durch nur punktuelle Aktivitäten zu ersetzen, wäre der Lan- 
desentwicklung vermutlich abträglich; erneut sei in diesem Kontext an die bis zum „Schlüsseljahr“ 2019 zu erbringenden organisationspolitischen Vorleistungen und die nach innen wie nach außen zu gewährleistenden Koordinationsaufgaben erinnert. Die Voraussetzungen für eine Fortsetzung des niedersächsischen Reformprozesses sind mithin nur unzureichend gegeben; dies gilt gleichermaßen für die nicht nur im Gefolge des Landtagswahlkampfs gewachsene politische Polarisierung, den dadurch verstellten Blick auf funktional unabweisbaren Handlungsbedarf und schließlich die horizontal wie vertikal fehlende Kooperationsbereitschaft der Akteure.

\section{Regionale Kooperationsräume als Bezugspunkt}

Um gleichwohl den wachsenden Verflechtungen zwischen den Teilräumen Niedersachsens und in diesen wiederum zwischen den einzelnen gebietskörperschaftlichen (wie privaten) Akteuren gerecht zu werden, ergänzte der Autor die umfassende indikatorengestützte und durch zahlreiche Gespräche im Land bestätigte Bestandaufnahme der niedersächsischen Kommunalstruktur um einen Ausweis großflächiger regionaler Verflechtungsräume, für die die drei norddeutschen Metropolregionen und der verbleibende Raum West- und Südwestniedersachsens ausgewählt wurden. In Rahmen dieser nachfolgend wiedergegebenen Zuschnitte (vgl. Abb. 1) haben sich im Verlauf der vergangenen Jahre Raumstrukturen und neue Akteurskonstellationen formiert, die für die territoriale Logik des staatlichen wie des kommunalen Handelns von beträchtlicher Bedeutung sind. Dabei stehen auf der sogenannten Makroebene zunächst die länderübergreifenden Bezugsräume Niedersachen-Hamburg und Niedersachsen-Bremen im Vordergrund, gefolgt von West- und Südwestniedersachsen sowie schließlich dem niedersächsischen Zentralraum und Südniedersachsen. Die nach dieser Logik zunächst untypisch erscheinende, wie aufgezeigt landespolitisch aber an Bedeutung gewinnende Kategorie „Küstenraum“ tritt hinzu.

Innerhalb dieser ,regionalen Kooperationsräume“ kam es dann in Ergänzung der betrachteten Metropolregionen und Verdichtungsräume zum Ausweis der hier jeweils erkennbaren Binnenstruktur, gefolgt von den sich damit wiederum für die Kommunalebene verbindenden Konsequenzen. So wurde die Analyse gleichsam deduktiv von der Makro- über die Meso- bis hin zur Mikroebene des politischadministrativen Handelns geführt und auf diesem Weg zunehmend konkretisiert. Damit sollte auch die kontroverse und häufig polarisierende Diskussion Metropolregion/Verdichtungsraum versus Fläche (und hier insbesondere ländlicher Raum) einer konstruktiv-umsetzungsorientierten Sichtweise zugeführt werden, 
die eine Berücksichtigung der künftig wichtiger werdenden Verflechtungs- und Interaktionsmuster erlaubt. So erfordert nicht zuletzt das Selbstverständnis von Metropolregionen als „großräumigen Verantwortungsgemeinschaften“ solidarische Binnenstrategien, die nur über funktionsfähige und leistungsfähige Kommunalstrukturen umsetzbar sein dürften. Auch folgte man damit komplementären wirtschaftlichen, sozialen und gesellschaftlichen Entwicklungen.

Abbildung 1: Regionale Kooperationsräume (Makroebene)

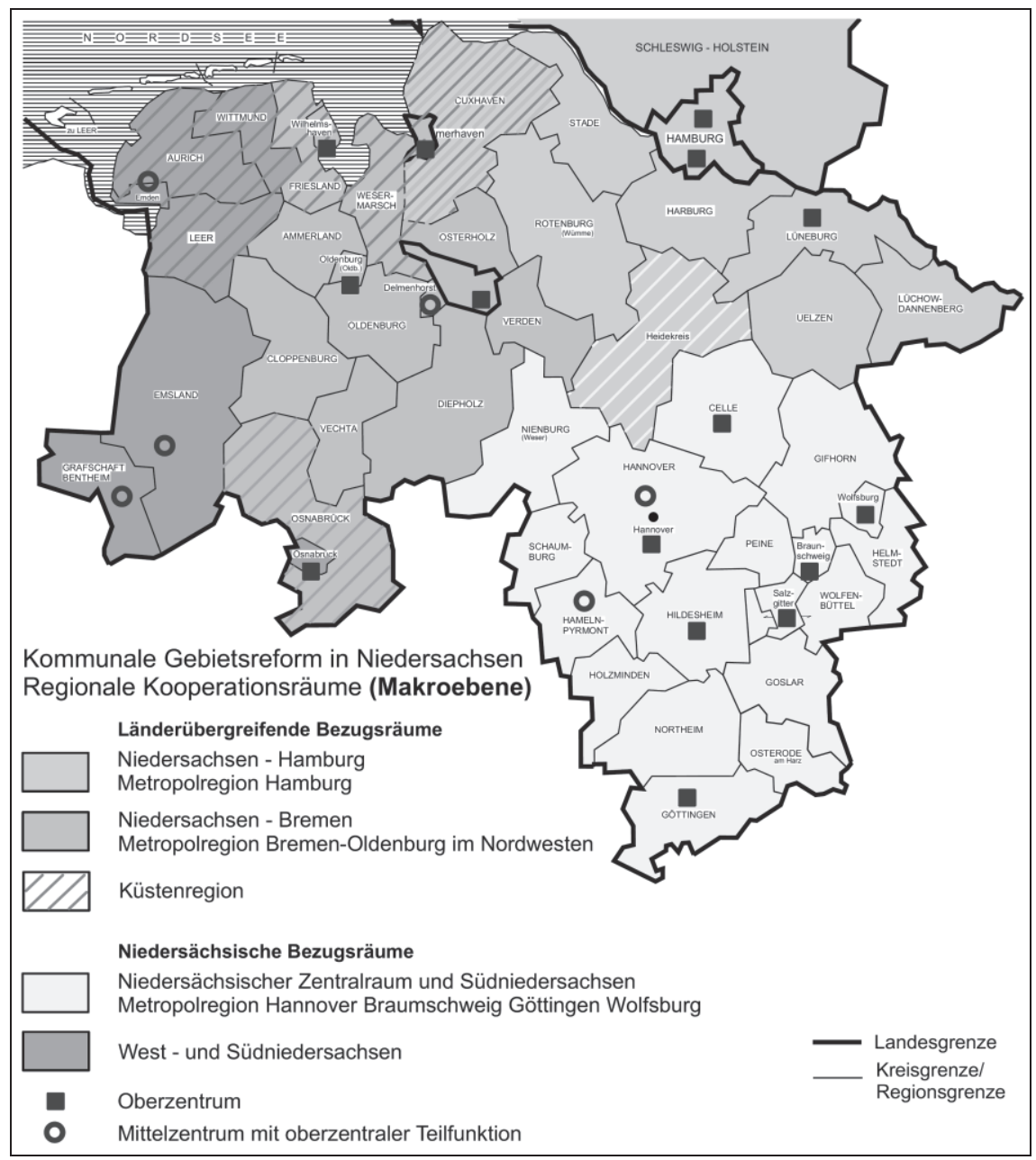

Anmerkung: Der Landkreis Osnabrück trat am 1. Juli 2010 der Metropolregion Bremen-Oldenburg bei, zudem ist der Heidekreis auch Mitglied der Metropolregion Hannover-Braunschweig-GöttingenWolfsburg; Quelle: Eigene Darstellung, Stand: November 2011. 
Im Ergebnis trifft die Analyse auf existente oder potentielle Regionaleinheiten wie Nordostniedersachsen, den Kommunalverband Niedersachsen-Bremen, das Oldenburger Land, das Regionalforum Bremerhaven, die Jadebay-Region Ostfriesland, die Strukturkonferenz Osnabrück, den erweiterten Wirtschaftsraum Hannover oder die Regionen Braunschweig, Göttingen und Weserbergland. Durch diese Verbindung von Makro-, Meso- und nachfolgender Mikrostruktur der Verflechtung wird die laufende „Regionalisierungs-Debatte“ gleichsam vom Kopf auf die Füße gestellt, die im Wesentlichen politisch geführte Diskussion funktional grundiert und kommt man dem tatsächlichen Bedarf und den sich daraus abzuleitenden Handlungsmöglichkeiten näher. So sind dann auch die zyklisch immer wiederkehrenden Forderungen nach einer „Großregion Braunschweig“ oder einer „Region Südniedersachsen“ hinsichtlich ihrer konkreten Handlungsoptionen wie Grenzen zu qualifizieren. Dabei erweist sich, dass von unrealistischen Regionsvorstellungen dann Abstand zu nehmen wäre, wenn sie, wie etwa im Fall Braunschweigs, Dimensionen umfassten, die kaum mehr arbeitsfähig und zudem selbstverwaltungsfeindlich sein dürften; sind sie darüber hinaus auch noch mit bereits verfassungsrechtlich auszuschließenden Vorstellungen verbunden (Abschaffung der Kreisebene), sollte dies bereits vorab Berücksichtigung (und Abwehr) finden.

\section{Stadt-Umland-Beziehungen: notwendige Systementscheidung}

Bei einer Verfolgung dieser Überlegungen gilt es zudem, für die städtischen wie ländlichen Räume Niedersachsens ein Kooperationsverhältnis herauszubilden, das handlungs- und zukunftsfähige Lösungen von Stadt-Umland-Beziehungen erlaubt. Das spannungsreiche, häufig konflikthafte Verhältnis zwischen den jeweiligen Zentralen Orten/Oberzentren und ihrem Umland bietet bekanntlich Anlass für langwierige Auseinandersetzungen, zudem erschwert es zukunftsfähige institutionelle Lösungen. Zwar wächst die Erkenntnis, dass die gegebenen siedlungsstrukturellen und ökonomischen Verflechtungen sowie die Folgen von Suburbanisierungsprozessen kooperative Handlungsmuster von Kernstadt und Umland nahe legen, doch steht dem meist eine nicht selten asymmetrische Nutzenverteilung von Kernstadt und Umland entgegen. Für die kommunalen Träger kommt es zu einem stetig wachsenden Unterhaltungsaufwand für öffentliche Einrichtungen, ohne dass dem die finanziellen Rahmenbedingungen entsprechend angepasst würden (die mehrfach gescheiterte Gemeindefinanzreform als ein Beispiel benannt); hinzu treten die Folgen des demographischen Wandels, 
der die Differenzen zwischen wie innerhalb der Regionen verstärkt und den interregionalen Wettbewerb erhöht.

In diesem Kontext erscheint die seitens der Städte/Oberzentren erhobene Forderung nach Kostenentlastung durch Beitragszahlungen der Umlandgemeinden angesichts der prekären Lage der Haushalte verständlich, zumal immer höheren Belastungen aus der Wahrnehmung zentralörtlicher Funktionen und überproportionaler Sozialausgaben ein Gewerbesteuer- und Einnahmenwachstum der angrenzenden Kommunen gegenüber steht. Gleichwohl vermochte sich der zentralörtliche Anspruch gegen die im Rahmen von Gebietsreformen aufgewerteten Umlandgemeinden kaum durchzusetzen, da deren Position durch die Selbstverwaltungsgarantie und die erwartbaren bzw. befürchteten politischen Kosten von Eingemeindungen gestützt wurde. Zwar beeinflussten neue Paradigma, etwa die Bildung der angesprochenen Metropolregionen, die Diskussion durchaus, ohne dass sich damit allerdings eine Lösung der Lastenausgleichsprobleme und der Verteilungskonflikte verband. Angesichts des auch operativ unzureichenden Gehalts der entsprechenden Diskussionen blieben Stadt-Umland-Probleme bis heute ein zentraler Gegenstand der staatlichen und kommunalen Verwaltungspolitik, wobei sich in der Praxis sechs Typen der Stadt-Umland-Organisation identifizieren lassen, die ein jeweils unterschiedliches Maß an materieller und institutioneller Integration aufweisen: Netzwerkartige Vereinigungen und Vereinbarungen; vertragliche Stadt-Umland-Kooperationen mit Träger- und Vollzugsfunktionen; gesetzliche Zuweisung von zentralörtlichen Schwerpunktbzw. vor-Ort-Aufgaben; Stadtregionale Verbände; Stadtregionale Verbände mit direkter gewählter Verbandsversammlung sowie Stadtregionale Gebietskörperschaften. Dieser letzte Typus stellt zugleich die am stärksten integrierte Kategorie von Stadt-Umland-Organisationen dar, wobei eine gebietskörperschaftliche Ebene entweder durch Eingemeindungen oder die institutionelle Verbindung mit der Kreisstufe inkorporiert wird. Das Spektrum entsprechender Lösungen reicht von der Eingliederung der Umlandgemeinden in den Zentralen Ort (als vollständige Eingemeidungen oder in Form einer Regionalstadt mit erweiterten Rechten der ehemals selbstständigen Randgemeinden) über Stadtkreise bzw. eine Stadtunion (Übernahme von Zuständigkeiten durch die Kernstadt mit einem direkt gewählten oder durch kommunale Delegierte gebildeten Repräsentativorgan) bis hin zur Konstruktion eines Regional- bzw. Großkreises, der die Kernstadt und die unmittelbar angrenzenden sowie ggf. auch die im weiteren Umfeld gelegenen Gemeinden zusammenfasst (die Region Hannover als Beispiel). 
Hält man an den Grundsätzen der deutschen Verwaltungsorganisation fest (Territorial- und Einwohnerprinzip, Trennung zwischen öffentlich- und privatrechtlicher Organisation, hierarchische Ordnung der Gebietskörperschaften) und orientiert man sich dabei an den skizzierten Organisationstypen, kommen für Niedersachsen vor allem zwei Vorgehensweisen in Betracht: die Auflösung einer einheitlichen Verbandsstruktur mit Kreisfunktion (verbunden mit der Überführung des Oberzentrums in eine kreisfreie Stadt und Eingliederung der sonstigen Mitgliedskommunen in umliegende Gemeindeverbände) und die Erbringung der dann vorwiegend ergänzenden und entwicklungspolitisch orientierten StadtUmland-Funktionen in mehr oder minder stark integrierten Kooperationszusammenhängen - oder aber die Beibehaltung und Fortentwicklung der gemeinsamen Kreisstruktur durch eine stärkere Integration von Kernstadt und Umlandgemeinden, was sowohl die Wahrnehmung gegebener Aufgaben und die Zuständigkeitsverteilung als auch das institutionelle Verhältnis der Mitgliedskommunen untereinander betrifft.

Angesichts dieser Ausgangssituation besteht eine Option darin, lediglich die vier unbestrittenen Großstädte als lebens-, handlungs- und zukunftsfähige Wachstumskerne auszuweisen und daneben kompetente Stadt-Umland-Kreise vorzusehen, die den gegebenen und wachsenden Verflechtungsprozessen folgen. Infolgedessen bedarf es keiner erneuten Diskussion von Hannover, Braunschweig, Osnabrück und Oldenburg, wohl aber einer Erörterung jener mittelgroßen und kleinen kreisfreien Städte, allen voran Wilhelmshaven, Delmenhorst und Emden. Göttingen stellt ein gewisses Sonderproblem insofern dar, als die angesprochene problematische Struktur Südniedersachsens ggf. eine untypische Organisationsform nahelegt. Gleichwohl wäre auch zu erwägen, die heterogene Stadtebene Niedersachsens zu öffnen, was dann die Kreisfreiheit von sich expansiv entwickelnden Städten, wie etwa Göttingen oder Lüneburg, vorsehen könnte. Allerdings zahlt das Umland dafür einen Preis, der sowohl in Südniedersachsen als auch in Nordostniedersachsen eine weitere „Verelendung“ des die Stadt umgrenzenden Raums mit sich bringen dürfte. Insofern präferiert der Autor eine Territorialstruktur, die außer den benannten vier Großstädten eher den Verflechtungsprozessen folgt und ihnen die Form erweiterter Kreisstrukturen gibt. Zwar sind die Begriffe „Großkreis“ und „Regionalkreis“ parteipolitisch besetzt, doch geht das dahinter stehende Denken funktional in die richtige Richtung. 


\section{Der ländliche Raum: von der Kooperation zur Fusion}

Aber auch für die eher ländlich strukturierten Räume Niedersachsens, die in Teilen ohne ein Oberzentrum auskommen, gilt, dass man sich der Diskussion um eine erweiterte interkommunale Kooperation (und etwaige territoriale Konse-

Tabelle 2: Räumliche und strategische Kooperationsvorteile

\begin{tabular}{|c|c|c|c|c|}
\hline $\begin{array}{l}\text { Raumbe- } \\
\text { zug }\end{array}$ & $\begin{array}{l}\text { Strategi- } \\
\text { sche Rele- } \\
\text { vanz }\end{array}$ & $\begin{array}{l}\text { Ermessens- } \\
\text { spielräume }\end{array}$ & $\begin{array}{l}\text { Beispiele } \\
\text { Aufgaben }\end{array}$ & Begründung \\
\hline \multirow{9}{*}{ Ortsbezug } & \multirow{3}{*}{ Hoch } & Hoch & \multirow{2}{*}{$\begin{array}{l}\text { Soziale u. Jugendhilfe- } \\
\text { einrichtungen }\end{array}$} & \multirow{4}{*}{$\begin{array}{l}\text { Immobiles Klientel } \\
\text { Örtliche Versorgungs- } \\
\text { funktion, Daseinsvorsorge } \\
\text { Gewährleistungsansprüche } \\
\text { an die Kommune }\end{array}$} \\
\hline & & Mittel & & \\
\hline & & Gering & \multirow{2}{*}{$\begin{array}{l}\text { Transferleistungen der } \\
\text { Jugendhilfe und nach } \\
\text { dem SGB II }\end{array}$} & \\
\hline & \multirow{3}{*}{ Mittel } & Hoch & & \\
\hline & & Mittel & \multirow{2}{*}{ Schulträgerschaft } & \multirow{2}{*}{$\begin{array}{l}\text { Häufig örtlich relevante } \\
\text { Gestaltungsmöglichkeiten }\end{array}$} \\
\hline & & Gering & & \\
\hline & \multirow{3}{*}{ Gering } & Hoch & \multirow{3}{*}{$\begin{array}{l}\text { Personen- und Melde- } \\
\text { wesen } \\
\text { Straßen- und Verkehrs- } \\
\text { Aufsicht }\end{array}$} & \multirow{3}{*}{$\begin{array}{l}\text { Lokale Nachfrage und } \\
\text { örtliches Vollzugserfor- } \\
\text { dernis } \\
\text { Geringe Gestaltungs-, aber } \\
\text { hohe Ordnungsrelevanz }\end{array}$} \\
\hline & & Mittel & & \\
\hline & & Gering & & \\
\hline \multirow{9}{*}{$\begin{array}{l}\text { Regional- } \\
\text { bezug }\end{array}$} & \multirow{3}{*}{ Hoch } & Hoch & \multirow{3}{*}{$\begin{array}{l}\text { Wirtschaftsförderung } \\
\text { Sonderordnungsauf- } \\
\text { gaben (Umwelt usw.) }\end{array}$} & \multirow{2}{*}{$\begin{array}{l}\text { Gestaltungsanspruch und } \\
\text { interregionaler Wettbe- } \\
\text { werb vs. Bündelungser- } \\
\text { fordernisse }\end{array}$} \\
\hline & & Mittel & & \\
\hline & & Gering & & \multirow{4}{*}{$\begin{array}{l}\text { Lokalbezüge und Vor-Ort- } \\
\text { Präsenz bei Ordnungsauf- } \\
\text { gaben - zugleich Ver- } \\
\text { bundvorteile von Koope- } \\
\text { rationen in spezialisierten } \\
\text { Prozessen }\end{array}$} \\
\hline & \multirow{3}{*}{ Mittel } & Hoch & \multirow{3}{*}{$\begin{array}{l}\text { Rettungsleitstellen } \\
\text { Zentrale und Quer- } \\
\text { schnittsaufgaben in }\end{array}$} & \\
\hline & & Mittel & & \\
\hline & & Gering & & \\
\hline & \multirow{3}{*}{ Gering } & Hoch & \multirow{3}{*}{$\begin{array}{l}\text { den Bereichen Käm- } \\
\text { merei, Haupt-, Organi- } \\
\text { sations- und Rechtsamt }\end{array}$} & \multirow{3}{*}{$\begin{array}{l}\text { Erhöhte Bedeutung für die } \\
\text { Eigenentwicklung - } \\
\text { zugleich Größen- und } \\
\text { Verbundvorteile bei } \\
\text { verwaltungsinternen } \\
\text { Querschnittsaufgaben }\end{array}$} \\
\hline & & Mittel & & \\
\hline & & Gering & & \\
\hline \multirow{6}{*}{$\begin{array}{l}\text { Ortsunab- } \\
\text { hängigkeit }\end{array}$} & & Hoch & \multirow[t]{3}{*}{ Bezügeabrechnung } & \multirow{6}{*}{$\begin{array}{l}\text { Geringes Erfordernis einer } \\
\text { Vor-Ort-Präsenz } \\
\text { Standardisierte Prozesse } \\
\text { und Verfahren mit gerin- } \\
\text { ger lokal auszufüllender } \\
\text { Gestaltungskompetenz } \\
\text { Reduzierte Bedeutung für } \\
\text { die kommunale Eigenent- } \\
\text { wicklung }\end{array}$} \\
\hline & Mittel & Mittel & & \\
\hline & & Gering & & \\
\hline & \multirow{3}{*}{ Gering } & Hoch & \multirow{3}{*}{$\begin{array}{l}\text { Ausbildungsplatzver- } \\
\text { mittlung }\end{array}$} & \\
\hline & & Mittel & & \\
\hline & & Gering & & \\
\hline
\end{tabular}

Quelle: ISE-Strukturberichterstattung. 
quenzen) stellen sollte. Sie zielte durch eine Bündelung der Kräfte und potentielle Formen erweiterter Arbeitsteilung auf eine Schöpfung von Synergien, die sich in fast jedem Teilraum des Landes identifizieren lassen. Sucht man daher nach Möglichkeiten, etwa das Leistungsspektrum von Kreisen beizubehalten und es künftig auch noch kostengünstiger zu gestalten, bieten sich jene Formen der Zusammenarbeit an, die der Autor mit zahlreichen Beispielen in einer gesonderten Untersuchung dokumentierte und die inzwischen auch die Aufmerksamkeit der Bundesebene gefunden haben. Wichtig wäre es, hier über die sich schrittweise verdichtende Trias Kommunikation - Kooperation - Koordination von eher unverbindlichen Formen der Zusammenarbeit zu zunehmend konkreten Kooperationen und schließlich verbindlichen Koordinationsformen zu gelangen, die bis hin zu Arbeitsteilungen zwischen den Gebietskörperschaften reichen. Das Potential hierfür ist trotz gegenteiliger Behauptungen niedersächsischer Akteure deutlich unterausgeschöpft, obwohl Beispiele aus anderen Flächenländern auf nachahmenswerte Entwicklungen verweisen.

Ein ernsthafteres Bemühen um interkommunale Zusammenarbeit (IKZ), besser: interkommunale Gemeinschaftsarbeit, hätte auch zur Kenntnis zu nehmen, dass die sich hierfür anbietenden Aufgabenfelder nahezu unbeschränkt sind (vgl. die Übersicht in Tab.2), zudem den schrittweisen Übergang in eine funktionsfähige Arbeitsteilung (die wiederum in eine spätere Fusion münden könnte) erlauben. Auch von daher sollte man von defensiven (und häufig eher exkulpativen) Selbstzuschreibungen (,machen wir doch schon lange“) Abstand nehmen und sich einer diesbezüglichen, in anderen Flächenländern längst dokumentierten Innovations- und Lernfähigkeit öffnen.

\section{Komplementäre Landespolitiken: Verbesserung der Ressortkoordina- tion, Ausbau der Regierungsvertretungen, aktive Grenzüberschreitung}

Die von der kommunalen Ebene abzufordernde Reformbereitschaft wäre schließlich durch komplementäre Verhaltensweisen der Landesregierung zu ergänzen. Hier findet sich zunächst das seit Jahren auch für Niedersachsen vorgetragene Desiderat einer deutlich verbesserten Ressortkoordination, die nach Abschaffung der Bezirksregierungen horizontal wie vertikal um ein aktiveres Abstimmungsverhalten zwischen den Akteuren bemüht sein sollte. Dies gilt sowohl für Querschnitts- wie für Sektoralpolitiken und auch nicht nur für den Prozess der Politikformulierung, sondern immer unter Einschluss des Vollzugs. Die Voraussetzungen für ein derart ergebnisorientiertes Handeln wurden mehrfach angesprochen, ihre Umsetzung steht allerdings noch aus. Damit begibt sich die jeweils amtie- 
rende Regierung auch der Chance, den Erfolg von Strukturreformen auf Landesebene (hier die Abschaffung der Bezirksregierungen) zu dokumentieren. Entsprechend zahlreich waren die Kritiken derer, die sich zwischenzeitlich des ,kurzen Weges“" nach Hannover beraubt sahen. Zwar spielen hier regionale und lokale (Sonder-)Interessen eine spezifische Rolle, doch verbleibt ein kommunikatives Vakuum, das auszufüllen sich anbietet.

In diesem Zusammenhang wären die in Oldenburg, Lüneburg und Braunschweig eingerichteten Regierungsvertretungen in ihrem Leistungsspektrum und ihrer Reichweite zu überprüfen. Der Autor hat dies mit seinem Evaluationsbericht zu diesen dislozierten Einheiten der Ministerialverwaltung getan, der unter der Überschrift „Aufbruch oder Abbruch“ eine beträchtliche interne Diskussion auslöste. Auch hier fand sich allerdings ein Umsetzungsdefizit insofern, als die Landesregierung keine der beiden Empfehlungen aufnahm und damit die Regierungsvertretungen in einer eher unbedeutenden Rolle des Informationsträgers beließ - statt sie zu den bei ihrer Begründung erhofften „Entwicklungsagenturen“ auszubauen. Die Opposition nahm dies im Wahlkampf zum Anlass, um sog. „Landesbeauftragte“ zu fordern, deren beschriebene Tätigkeit sich in Teilen als wortidentisch mit dem Evaluationsbericht erwies.

Schließlich bietet es sich an, die in zahlreichen öffentlichen Erklärungen als wichtig bezeichneten „Grenzüberschreitungen“ (lokal, regional und national) auch mit handlungsorientierten Politikentwürfen zu verbinden. Dies sollte nicht nur für Gemeinde- oder Kreis-Kooperationen, sondern auch für eine erweiterte Länder-Länder-Zusammenarbeit gelten, zumindest da, wo wachsende Verflechtungsbeziehungen dies ohnehin nahe legen. Beispiele hierfür finden sich in nahezu allen Landesteilen, vom Eichsfeld über das Weserbergland oder die Unterelbe bis in den Grenzraum zu den Niederlanden. Ein solch erweitertes Verständnis bietet sich zudem mit Blick auf die veränderte EU-Förderung an; sie macht landesinterne Bündelungsprozesse unausweichlich.

\section{Handlungspotentiale und Grenzen kommunaler Selbst- verwaltung}

In dem diesen Bericht abschließenden Kapitel sollen die angesichts der niedersächsischen Ausgangssituation möglichen Handlungsoptionen nun zusammengefasst dargestellt werden. Dies beinhaltet einen Blick auf die Akteure und die Gestaltung des Reformprozesses, summiert die sich bietenden Ansätze und stellt die zu erwartenden materielle Ergebnisse textlich wie kartographisch dar. 


\section{Zur Gestaltung des Reformprozesses: kein Erkenntnis-, sondern ein Entscheidungs- und Vollzugsdefizit}

Zunächst freilich ist festzuhalten, dass die weiteren Stufen des Reformprozesses weniger durch die Suche nach neuen/erweiterten Erkenntnissen und auch nicht durch weitere Expertengremien, Enquete-Kommissionen u.a.m. geprägt sein sollten, sondern durch ein explizites (und, mit Verlaub, mutiges) politisches Handeln. Nachdem die vergangene Legislaturperiode unter das Prinzip lediglich freiwilliger Kooperationen und Fusionen im Kommunalbereich gestellt wurde, erscheint es jetzt an der Zeit, den sich mit der Landesverfassung verbindenden politischen Gestaltungsauftrag wahrzunehmen und ein umsetzbares Handlungskonzept zu erarbeiten. Angesichts der gegeben Informationsbasis geht es dabei weniger um neue „Prüfaufträge“, sondern eher um die Sichtung der durchwegs vorliegenden empirisch-analytischen Erkenntnisse und eine sich anschließende Willensbildung und Entscheidung. Natürlich ist die Aufgabe komplex, da die mehrfach angesprochene Heterogenität des Landes gleichförmige Vorgehensweisen erschwert, doch findet sich für jeden Teilraum Niedersachsens durchaus ausreichendes quantitatives wie qualitatives Material, das belastbare prognostische Aussagen und nachfolgende Handlungsempfehlungen zulässt. Im Ergebnis bietet sich ein eher pragmatisches denn an „Visionen“ ausgerichtetes Vorgehen an. So dokumentiert die in Oppositionskreisen erörterte Aufteilung Niedersachsens in acht bis etwa zwölf Regionen erkennbar Wunschdenken und bleiben Großkreise jenseits der 400.000 Einwohner-Grenze schon deshalb kaum vermittelbar, weil damit funktional vertretbare Größenordnungen verletzt und das Recht auf Selbstverwaltung eingeschränkt sein dürften. Zudem stehen Vorstellungen wie die nach Bildung ,,verfasster Regionen“ unter dem Verdikt, zu Heterogenes aufeinander beziehen $\mathrm{zu}$ wollen, auch wird übersehen, dass der Fall „Region Hannover“ nach aller vorliegenden Erkenntnis ein Solitär bleiben dürfte. Stattdessen empfiehlt es sich, an der gewachsenen gebietskörperschaftlichen Struktur anzusetzen und über die punktuelle Verschmelzung wenig lebensfähiger Kreise und nur noch eingeschränkt selbstverwaltungsfähiger Gemeinden eine zukunftsfähige Kommunalstruktur zu schaffen. Dass dabei der Stand der Rechtsprechung des Bundes- und der Landesverfassungsgerichte zum Erhalt der Selbstverwaltung zu berücksichtigen ist, versteht sich von selbst. 


\section{Die Akteure: mangelnde Lernfähigkeit, begrenzte Handlungsorientie- rung, Flucht aus der Verantwortung}

Als ein auffälliges Merkmal bei der Erörterung handlungs- und zukunftsfähiger Kommunalstrukturen in Niedersachsen erweist sich trotz großer Diskussionsbereitschaft die in Teilen eingeschränkte Lernfähigkeit der Akteure, wobei dieser Begriff sich nicht nur auf die Vertreter von Städten, Kreisen und Gemeinden, sondern auch auf Repräsentanten der Parteien, der Verbände und der zuständigen Einrichtungen des Landes richtet. Hier wird erkennbar, dass man sich zwischenzeitlich zur Sicherung des jeweiligen Besitzstandes gleichsam „vergraben“ hat und Gesprächen zwar nicht ausweicht, sie aber selten handlungs- oder gar veränderungsbereit führt. Von daher erscheint es dringlich, dass die bereits angesprochene Trias von verstärkter grenzüberschreitender Kommunikation, nachfolgender Kooperation und verlässlicher Koordination in den kommenden Monaten und Jahren genutzt wird, um - dem Beispiel anderer Flächenländer folgend - die kommunale Ebene ,zukunftsfest“ zu machen. So verwundert insbesondere das Festhalten an in Teilen nicht mehr nachgefragten und/oder finanzierbaren Formen der Daseinsvorsorge, verwechselt man gelegentlich das eigene Interesse mit dem der Bürgerschaft und fehlt es im Rahmen zahlreicher punktueller Vereinbarungen an materieller Konkretion und terminlicher wie finanzieller Verbindlichkeit. So bleiben Zielvereinbarungen häufig Absichtserklärungen, „flexibilisiert“ man zeitliche Vereinbarungen und werden materielle Handlungsschwerpunkte umgedeutet oder doch zumindest uminterpretiert. All dies ist aufgrund unterschiedlicher Interessenlagen gewiss verständlich, lähmt aber seit geraumer Zeit einen Reformprozess, der aufgrund der unbestreitbaren Datenlage und belastbarer Selbstauskünfte unverzichtbar sein dürfte. Daran zu erinnern verletzt nicht die (notwendigerweise zurückhaltende) Rolle eines anwendungsorientiert arbeitenden Wissenschaftlers, sondern ist Ausdruck der Verpflichtung, auf die Konsequenzen eines zu langen „Aussitzens“ unabweisbarer Reformen zu verweisen.

Im Gefolge der angesprochenen Verhaltensmuster droht Niedersachsen im Vergleich zu den anderen deutschen Flächenländern zurückzufallen. Zwar gilt das Land noch immer, gemeinsam mit Baden-Württemberg, als verwaltungspolitisch „führend“, da die Abschaffung der Bezirksregierungen und der damit verbundene konsequente Übergang zur administrativen Zweistufigkeit als gleichsam modellhaft anerkannt wird (in Baden-Württemberg richtete sich der dort verfolgte Reformansatz auf die Auflösung aller Sonderbehörden und die Übertragung der verbleibenden Aufgaben in die Regierungspräsidien bzw. die Landkreise), doch mehren sich die Vorbehalte, nach denen der Reformansatz „einseitig“ blieb, eine 
komplementäre (und ergebnisorientierte) Überprüfung der kommunalen Ebene bis heute nicht wirklich erkennbar sei, weder unter christdemokratischer noch unter sozialdemokratischer Führung. Insofern bietet es sich an, dieses Defizit im Verlauf der kommenden Legislaturperiode aufzulösen, wobei Niedersachsen von den bereits vollzogenen oder ablaufenden Kommunalreformen in den anderen Flächenländern durchaus lernen könnte. Zwar ist auch hier die Unterschiedlichkeit der Länder zu beachten, doch finden sich gerade mit Blick auf „optimale Betriebsgrößen“ für Kreise, kreisfreie Städte und Gemeinden zahlreiche neue und für Niedersachsen einsetzbare Erkenntnisse. Es geht mithin um einen konsistenten Reformansatz, der Unterschiede nicht künstlich einzuebnen sucht, sondern sie veränderten Territorialstrukturen zuführt, vor allem dann, wenn die Handlungsfähigkeit deutlich beeinträchtigt ist und die Zukunftsfähigkeit durchaus „verspielt“ zu werden droht. So wie für die Fortentwicklung des deutschen Föderalismus Argumente der Leistungs- mit denen der Verteilungsgerechtigkeit zu verbinden sind, stellt sich auch für die kommunale Ebene die Aufgabe, Selbstverwaltung innerhalb handlungsfähiger struktureller Voraussetzungen auch zu gewährleisten. Dass dabei die wachsende Internationalisierung des Regierungsund Verwaltungshandelns zu berücksichtigen ist, sollte sich von selbst verstehen - nicht als wohlfeile Absichtserklärung, sondern als selbstbewusste Präsenz nach außen und eine den Namen verdienende Bündelung von Landespolitiken nach innen. Der Wettbewerb um leistungs- und zukunftsfähige Territorialstrukturen ist auch im öffentlichen Bereich längst eröffnet.

\section{Grundlegende Handlungsoptionen}

Sucht man vor diesem Hintergrund die sich bietenden Handlungsoptionen zu systematisieren, so kann zwischen einer Optimierung des Status quo, einer erweiterten interkommunalen Kooperation, punktuellen Anpassungen und selektiven Gebietsreformen sowie schließlich einem Gesamtansatz unterschieden werden, der eine breitere regionale Orientierung und erhöhte Flexibilität nahe legt. Die daraus letztlich folgenden Konsequenzen für die gebietskörperschaftliche Struktur (Fusionen, Einkreisungen, Bildungen von „Großkreisen“) werden abschließend zusammenfassend bewertet und in ihren Vor- wie Nachteilen gegenübergestellt.

Allen ausgewiesenen Handlungsmöglichkeiten liegt zugrunde, dass sich mit Blick auf die niedersächsische Kommunalstruktur Optimierungspotential und Rationalitätsreserven erkennen lassen, die, vor allem, aber nicht nur auf Effizienz- und Effektivitätserwägungen gestützt, in der Summe einen Eingriff in be- 
stehende Verwaltungsstrukturen auf der kommunalen Ebene rechtfertigen. Dabei bleibt die Verhältnismäßigkeit von Mitteleinsatz und Ertrag etwaiger Reformen zu beachten. Den wesentlichen Bezugspunkt bildet die Wahrung von Bürgernähe, Identität und demokratischer Teilhabe, hier als örtliche und politische Integrationsfähigkeit kommunaler Gebietskörperschaften verstanden. Darauf bezog sich auch das Landesverfassungsgericht Mecklenburg-Vorpommern in seinem ablehnenden Urteil zum ersten Ansatz der dort vorgesehenen Kreisgebietsreform. Darin wurde unter Berufung auf die Rechtsprechung des Bundesverfassungsgerichts deutlich geltend gemacht, dass dem benannten Kriterium (insbesondere dem ,demokratischen Gesichtspunkt der örtlichen Teilhabe der Bürgerschaft an der Erledigung ihrer öffentlichen Aufgaben“) nicht nur erhebliche, sondern gegenüber reinen Wirtschaftlichkeitserwägungen sogar superiore Bedeutung zukommt. Obgleich diese strikte Auslegung unter funktionalen und staatspraktischen Gesichtspunkten durchaus diskussionswürdig erscheint, verbinden sich mit ihr doch gewichtige Implikationen. Dies gilt vor allem dann, wenn in Aussicht genommene Reorganisationsprozesse gesetzlich verfügte Territorialreformen einschließen und den Bestand gegebener Gebietskörperschaften berühren. Das dabei herangezogene öffentliche Wohl und die sich daraus ableitenden gesamtstaatlichen Interessen bedürfen deshalb einer materiellen wie im Verfahren überzeugenden Begründung. Berücksichtigt man die auch in Niedersachsen nicht auszuschließende Möglichkeit einer verfassungsgerichtlichen Auseinandersetzung, ergeben sich wenigstens vier Anforderungen an den Prozess und die argumentative Absicherung von Reorganisationsprozessen:

- Die in Betracht gezogenen Maßnahmen (Einkreisungen, Gemeinde- und Kreisfusionen usw.) müssen stichhaltig begründet und im Ergebnis in erheblichem Umfang dazu geeignet sein, die zugrunde liegenden Erfordernisse des öffentlichen Wohls zu erfüllen.

- Dies impliziert, ex ante unterschiedliche Handlungsansätze ergebnisoffen in Betracht zu ziehen und sorgfältig gegeneinander abzuwägen, wobei auch solche Alternativen einzubeziehen sind, die den Bestand vorhandener kommunaler Gebietskörperschaften nicht in Frage stellen.

- Ferner müssen die eine Reform veranlassende Ausgangslage, darauf bezogene Maßnahmen und ihre Effekte in einem angemessenen Verhältnis zueinander stehen, wobei dem Erhalt der demokratischen, örtlichen und politischen Integrationsfähigkeit besonderes Gewicht zukommt. Jenseits der (mit Hilfe von Alternativen) festzustellenden Notwendigkeit von Struktureingriffen verlangt dies, dass jeder einzelne Schritt einen wesentlichen Beitrag da$\mathrm{zu}$ leisten muss, die avisierten Ziele zu erreichen. 
- Schließlich sind die benannten Analyse- und Entscheidungsschritte in ein geordnetes Verfahren einzubetten, dessen Ausgang nicht über bestimmte Prämissen von vornherein determiniert sein darf.

Während im vorliegenden Fall die Situationsanalyse in ausreichendem Maße zu begründen vermochte, dass eine Überprüfung der niedersächsischen Kommunalstrukturen Reformansätze nicht nur sinnvoll erscheinen lässt, sie in Teilen vielmehr erforderlich macht, müssen die in Reaktion hierauf zu entwickelnden Reformoptionen deshalb alle (oder doch zumindest viele) denkbaren Handlungsalternativen einschließen und in Form konkreter Modelle auf ihre reformimmanenten Wirkungen hin überprüft werden. Diese Arbeitsschritte bilden mithin einen wesentlichen Teil des Reformprozesses, erlauben sie im Ergebnis doch erst die Beantwortung der Frage, ob materiell sinnvolle Reorganisationsmaßnahmen verfügbar, praktikabel und politisch durchsetzbar sind, was wiederum ganz wesentlich deren rechtliche Zulässigkeit im Hinblick auf ihre Verhältnismäßigkeit bedingt. Quantitative Relationen und allgemeine Entwicklungsgrundsätze ohne konkrete Hinweise auf deren Umsetzung reichen hier nicht aus und könnten sich später als Hindernis für eine belastbare Entscheidungs- und Kompromissfindung herausstellen; entsprechend ausgerichtete Expertisen bleiben deshalb notwendigerweise unvollständig.

Angesichts dieser rechtlichen und politischen Bedingungen für eine Strukturreform erscheint es angezeigt, möglichst gesamthaft die zur Verfügung stehenden Handlungsansätze zu erfassen und diese ggf. zu praxistauglichen Reformmodellen für einzelne Regionen, hier als Regionale Kooperationsräume verstanden, zu verdichten. Im Nachfolgenden wird einer deduktiven Logik gefolgt, die jeden Entwicklungsschritt argumentativ und mit Blick auf die vorgefundene Empirie im Land abzusichern sucht.

Grundsätzlich sind bei dem erkennbaren und hier umfassend dokumentierten Reformbedarf zwei Vorgehensweisen denkbar:

- Die erste geht den kommunalverfassungsrechtlich vollständig risikolosen Weg einer Optimierung unter Wahrung der gegebenen Verwaltungsstrukturen.

- Die zweite Option besteht in Ansätzen, die Synergien durch die benannten Größenersparnisse und Verbundvorteile zu erreichen, was jeweils im Einzelfall den Einbezug von wenigstens zwei Gebietskörperschaften erforderlich macht. 
Tabelle 3: Handlungsansätze zur Reorganisation der kommunalen Kreisstufe

\begin{tabular}{|c|c|c|c|}
\hline & \multirow{2}{*}{$\begin{array}{l}\text { Keine Änderung } \\
\text { vorhandener } \\
\text { Gebietsstrukturen }\end{array}$} & \multicolumn{2}{|c|}{ Änderung vorhandener Gebietsstrukturen } \\
\hline & & $\begin{array}{l}\text { Keine Auflösung und/oder } \\
\text { Neubildung bestehender } \\
\text { Gebietskörperschaften }\end{array}$ & $\begin{array}{l}\text { Auflösung und/oder } \\
\text { Neubildung bestehender } \\
\text { Gebietskörperschaften }\end{array}$ \\
\hline \multirow{2}{*}{$\begin{array}{c}\text { Freiwillige } \\
\text { Veränderung } \\
\text { (auf vertraglicher } \\
\text { Grundlage) }\end{array}$} & \multirow{2}{*}{$\begin{array}{c}\text { Interkommunale } \\
\text { Zusammenarbeit } \\
\text { zwischen Kreisen } \\
\text { und/oder kreisfreien } \\
\text { Städten }\end{array}$} & $\begin{array}{l}\text { Freiwillige Einkreisungen } \\
\text { kreisfreier Städte }\end{array}$ & $\begin{array}{l}\text { Freiwillige Fusion/Neubildung } \\
\text { von Kreisen* }\end{array}$ \\
\hline & & $\begin{array}{l}\text { Freiwillige Gebietsänderungen/ } \\
\text {-anpassungen von Kreisen } \\
\text { und/oder kreisfreien Städten }\end{array}$ & $\begin{array}{c}\text { Freiwilliger von der bisherigen } \\
\text { Gebietskulisse abweichende(r) } \\
\text { Neuzuschnitt/Neubildung } \\
\text { von Kreisen }\end{array}$ \\
\hline \multirow{2}{*}{$\begin{array}{l}\text { Zwangsweise } \\
\text { Veränderung } \\
\text { (per Gesetz oder } \\
\text { Verordnung) }\end{array}$} & \multirow{2}{*}{$\begin{array}{l}\text { Strukturierte/gesteuerte } \\
\text { Kooperation zwischen } \\
\text { Kreisen und/oder } \\
\text { kreisfreien Städten }\end{array}$} & Einkreisungen kreisfreier Städte & Fusion/Neubildung von Kreisen* \\
\hline & & $\begin{array}{c}\text { Gebietsänderungen/ } \\
\text {-anpassungen kreisfreier Städte }\end{array}$ & $\begin{array}{l}\text { Von der bisherigen Gebietskulisse } \\
\text { abweichende(r) Neuzuschnitt/ } \\
\text { Neubildung von Kreisen }\end{array}$ \\
\hline
\end{tabular}

* Ggf. unter Einschluss kreisfreier Städte

Quelle: Eigene Darstellung nach der ISE-Strukturberichterstattung.

Als Konzentrationsansätze können dabei alle Lösungen verstanden werden, die auf eine Zusammenfassung von Zuständigkeiten und Ressourcen zielen und insgesamt wie im Einzelfall mehr als eine bestehende kommunale Gebietskörperschaft einbeziehen. Die rechtliche Relevanz und erkennbare Empirie vorangegangener Gebiets- und Verwaltungsreformen in den Flächenländern legen dabei eine Unterscheidung danach nahe, ob

- die in Rede stehenden Konzentrationsmaßnahmen die gegebenen Gebietsstrukturen in Frage stellen und

- $\quad$ sie freiwillig oder zwangsweise (also auf dem Gesetzes- bzw. Verordnungswege) zu vollziehen sind.

Die obige Darstellung systematisiert die sich daraus ergebenden Handlungsansätze, wobei im Fall der für Gebietsstrukturen relevanten Maßnahmen noch einmal zwischen solchen Reformen unterschieden wird, die nicht nur die Gebietsstrukturen, sondern auch den Bestand vorhandener Gebietsstrukturen betreffen, sowie jenen, die diese im Kern weiterhin unberührt lassen. 
Die ausgewiesenen Ansätze sind nicht notwendigerweise alternativ, sondern durchaus komplementär nutzbar, etwa mit Blick auf die Verbindung von Neugliederungen und Kooperationen. Trotz der unbestreitbaren Vorteile größerer Einheiten sind allerdings Wirkungsgrenzen zu beachten. Dabei handelt es sich im Wesentlichen um (dauerhafte) Reformnachteile, insbesondere Transaktions- und die bereits mehrfach angeführten Demokratiekosten; im Fall von Gemeinschaftsarbeit kommen Verzögerungen und die Zurückhaltung eines Autonomiebesatzes hinzu. Im Ergebnis legen entsprechende Erfahrungen und Plausibilitätsbetrachtungen nahe, dass Entwicklungs-, Verbund- und Größenvorteile mit der Ausdehnung und Einwohnerzahl des gebietskörperschaftlichen Zusammenwirkens nicht endlos anwachsen, sondern ihr Ertrag ab einem gewissen Punkt wieder sinkt, da die angesprochenen Defizite in Abhängigkeit von der Komplexität, der Vielzahl und dem Umfang größerer Einheiten steigen; je nach Gewichtung (insbesondere der dargestellten Demokratiekosten) kann dies trotz erheblicher Synergien auch in eine gegenüber dem status quo negative Nutzenbilanz münden. Hieraus folgt, dass es für jeden Handlungsansatz wie auch für die Kombination unterschiedlicher Maßnahmen so etwas wie ein Optimum und, daraus abgeleitet, einen sinnvollen ,Reformkorridor" gibt, innerhalb dessen die Konzentrationseffekte von Reorganisationsmaßnahmen hinreichend groß ausfallen und Aufwand, Kosten wie Ertrag in einem angemessenen Verhältnis zueinander stehen, mithin die Abbildung 2: Konzentrationseffekte bei Neugliederungen/Fusionen

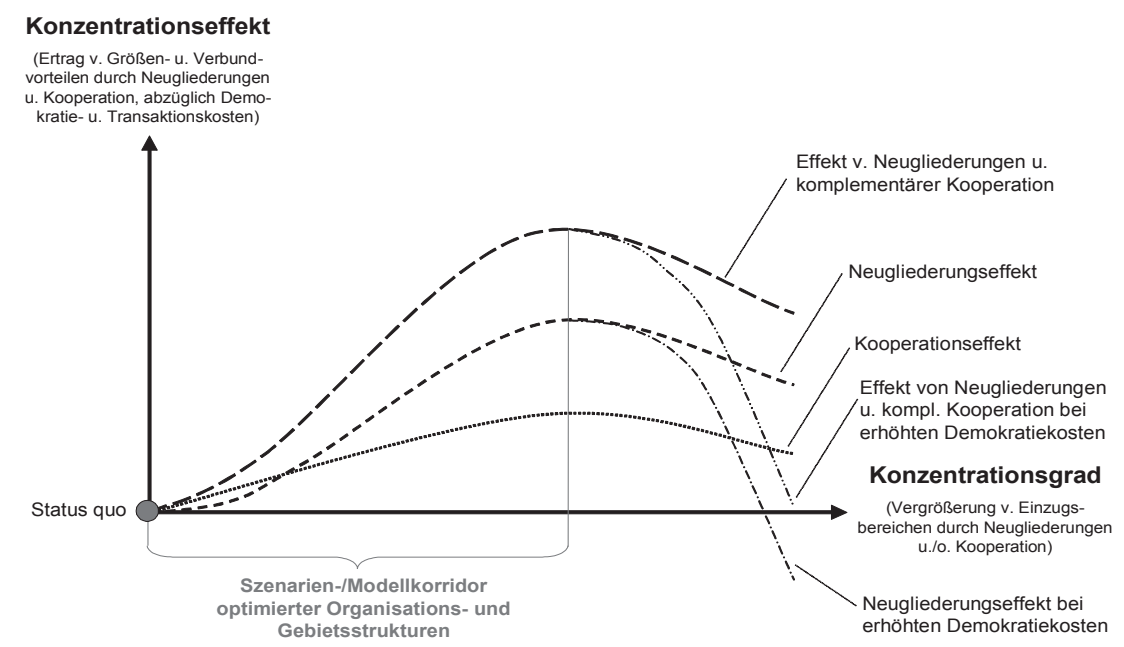

Quelle: Eigene Darstellung nach der ISE-Strukturberichterstattung. 
normativen, funktionalen und politischen Anforderungen an eine Verwaltungsstrukturreform erfüllt werden. Diese Zusammenhänge sind in der folgenden Abbildung graphisch dokumentiert und wurden in einer früheren Untersuchung vom Autor handlungsleitend eingesetzt.

Der Ausweis von Handlungsoptionen setzt an diesen Überlegungen an und sucht von einer Minimal- bis hin zu einer Maximallösung unterschiedliche Handlungsoptionen auszuweisen. Erstere beinhaltet mit Blick auf eine ergebnisoffene Prüfung von Alternativen die (bloße) Optimierung des status quo, die keiner Neugliederung/Fusion bedarf und zudem ohne größere staatliche Einflussnahme auskommt. Um dennoch auch hier Konzentrationswirkungen zu erzielen, kommt es dabei meist zu einer verstärkten interkommunalen Zusammenarbeit, die durch Förder- und Anreizpolitiken des Landes begünstigt wird, etwa in der Form einer landesseitigen Begleitung, die auf geordnete und strategisch ausgerichtete Formen der Gemeinschaftsarbeit achtet, damit gebildete Kooperationen zu einem späteren Zeitpunkt etwaigen Fusionen und Einkreisungen nicht widersprechen.

Das Maximalszenario hingegen sucht den höchsten Effekt von Verbund- und Größenvorteilen sowohl in fiskalischer als auch in entwicklungspolitischer Hinsicht zu verwirklichen. Als Orientierung bieten sich dafür regionalplanerische Zusammenhänge und Dimensionen an, die nachhaltige Eingriffe nicht nur nicht ausschließen, sondern auch befördern, bis hin zur Bildung von ausreichend integrationsfähigen und stabilen Kreisen. Im Ergebnis könnte das zu Größenordnungen führen, die Bevölkerungs- und Flächenzahlen umfassen, die im Bundesdurchschnitt als überdurchschnittlich sowie im Sinne der diskutierten normativen Anforderungen als Groß-oder Regionalkreise zu charakterisieren wären. Wiewohl dies auch auf der Grundlage des Urteils des Landesverfassungsgerichts Mecklenburg-Vorpommern nicht von vornherein als unzulässig, sondern ,nur“ als in besonderem Maße begründungsbedürftig einzuschätzen ist, stellen Regionalkreise doch einen Grenzfall dar, dessen weitere Ausdehnung normativ wie funktional kaum noch vertretbar erscheint. Gleichwohl bleibt der Einbezug solcher Vorstellungen sinnvoll und notwendig, um angesichts des dokumentierten Stabilisierungsbedarfs dem Anspruch einer ggf. erheblichen Strukturverbesserung gerecht werden zu können und zugleich einen Referenzpunkt für die Bewertung, Umsetzung und auch (spätere) Weiterentwicklung niederschwelligerer Lösungen aufzustellen.

Zwischen einer solcher Minimal- und Maximallösung kommt es dann zu weiteren Ausdifferenzierungen, die etwa als punktuelle Anpassung oder Reform mittlerer Reichweite zu bezeichnen sind. Beide beinhalten eine Fortsetzung jener im 
Rahmen der Optimierung des status quo angelegten Kooperationspolitiken, soweit diese nicht durch eine gleichzeitige Neugliederung aufgehoben bzw. überkompensiert werden. Die „Reform mittlerer Reichweite“ könnte hier noch einen Schritt weiter gehen und die Lösung von „Problemfällen“ mit einer erweiterten Anpassung auch in anderen Landesteilen verbinden. Dahinter stünde die Überlegung, flächendeckend die mit einer Neugliederung in Aussicht gestellten Effizienzrenditen zu erwirtschaften und zugleich ausgewogene Kommunalstrukturen im gesamten Land zu bilden. Mit den vier Optionen wird im Ergebnis nicht nur die erkennbare Empirie und der ihr folgenden Stabilisierungs- und Handlungsbedarf zusammengefasst, sondern auch ein prüf- und handlungsfähiges Kontinuum entwickelt, das dann nachfolgend weiter konkretisiert werden kann. Wichtig ist dabei, dass die aufgezeigten Konstellationen zwar unterschiedliche Handlungsansätze beinhalten, sich jedoch so ausgestalten lassen, dass sie untereinander anschlussfähig bleiben, eine wesentliche Voraussetzung dafür, im Rahmen eines sich anbietenden Konzentrationsprozesses effektive Freiwilligkeitsphasen vorzusehen und die darauf fußende Organisationsentwicklung nicht zuletzt rechtlich zulässig und vor allem konsensfähig zu gestalten.

\section{Erwartbare Ergebnisse: zukunftsfähige niedersächsische Kommunal- strukturen}

In der materiellen Zusammenfassung und bei Übertragung auf die niedersächsischen Verhältnisse soll schließlich zwischen einer „Minimallösung“, die sich im Wesentlichen auf Veränderungen der Kommunalstruktur in den erkennbaren Problemräumen des Landes richtet, einer „Entwicklungsvariante“, die ein Handlungsprogramm für die bevorstehende Legislaturperiode beinhaltet, und einem „Zukunftsmodell“, das einen weiteren Zeitraum umfasst, unterschieden werden. Letzteres könnte allerdings schneller Realität werden, als es dem Land und seinen Kommunalvertretern lieb ist. Zumindest gilt dies für den Fall, dass es im Vorfeld des Jahres 2019 zu einer umfassenden Überprüfung der Regierungs- und Verwaltungsorganisation der Bundesrepublik, unter Einschluss des kommunalen Bereichs, kommt. Knapper werdende öffentliche Ressourcen, beträchtliche Veränderungen der Bevölkerungsstruktur und wachsende transnationale Verpflichtungen werden schnellere und zukunftsorientiertere Entscheidungen erzwingen, von denen sich keine der gebietskörperschaftlichen Ebenen ausnehmen kann. 


\section{a. Eine erste landesweite Zusammenfassung}

Die ergebnisorientierte Darstellung der Erkenntnisse des zweimal fortgeschriebenen „Grundgutachtens“ erfolgt zunächst nicht über einen detaillierten Ausweis größerräumiger Optionen, sondern eher in Form einer „Punktation“ zu berücksichtigender Entwicklungen. Der Grad der Konkretisierung ist dabei unterschiedlich: Während für die drei Teil- (und Problem)regionen Südniedersachsen, Wolfsburg-Gifhorn-Helmstedt und Nordostniedersachsen bereits sehr konkrete Handlungsoptionen samt der mit ihnen verbundenen Konsequenzen vorgestellt werden, verbleibt es für die Situation in anderen Landesteilen bei Hinweisen auf die sich vor allem angesichts der Datenlage anbietenden Veränderungen. Die Ausführungen stützen sich dabei auf unterschiedliche Quellen: die umfassende indikatorengestützte Ausgangsanalyse, die zwischenzeitlich in fast allen Landesteilen geführten vor-Ort-Gespräche, weitere Kontakte mit politischadministrativen Funktionsträgern (auf Landes- und kommunaler Ebene) sowie ergänzende Informationen aus der Wirtschaft, den Verbänden, den Medien und der Fachöffentlichkeit. Zum Abschluss findet sich eine Kartierung der Handlungsempfehlungen.

\section{Südniedersachsen}

Auf dieser Basis ergeben sich für das von besonderen Strukturschwächen geprägte Südniedersachsen (Landkreise Göttingen, Northeim und Osterode a.H.) drei Optionen: Zum einen die nicht zuletzt aufgrund der Kommunalwahlergebnisse mögliche Bildung eines „Großkreises Südniedersachsen“, der eine Fusion aller drei Landkreise vorsieht; zum zweiten einen Handlungsansatz, der die Stadt Göttingen in die Kreisfreiheit „entlässt““ und den verbleibenden Kreis Göttingen mit den Kreisen Northeim und Osterode zusammenführt; sowie schließlich drittens das vom Autor in seiner teilregionalen Untersuchung präferierte Stufenmodell, das zunächst, nicht zuletzt Anregungen der handelnden Akteure folgend, eine Fusion von Northeim und Osterode vorsah und eine etwaige ,große Lösung“ („Triangel“-Option) erst nach einer tragfähigen Klärung des Verhältnisses von Stadt und Landkreis Göttingen einräumte. Heute stellt sich aufgrund unüberbrückbar erscheinender Interessendivergenzen zwischen den (parteipolitisch weitgehend homogenen) Akteuren eher die Frage eines Zusammengehens der Landkreise Göttingen und Osterode (inzwischen vollzogen), da sich Northeim mit Blick auf die Standorte dislozierter Verwaltungseinrichtungen (in einem etwaigen Großkreis) unzureichend gewürdigt sieht. Hinzu tritt, dass die finanziellen Vereinbarungen zwischen der Stadt und dem Landkreis Göttingen zu keinem dem Oberzentrum angemessen erscheinenden Ergebnis führten, sondern 
seitens der Stadt als drohende Fortsetzung einer ihre Entwicklungsdynamik beeinträchtigenden Lösung interpretiert werden. Hier gewinnen jene Stimmen an Gewicht, die der Stadt Göttingen die Kreisfreiheit zusprechen wollen. In logischer Reaktion auf diese Entwicklung wäre es letztlich auch denkbar, dem kreisseitig mit der Bildung eines „Kragenkreises“ der drei angesprochenen Gebietskörperschaften zu begegnen.

Hinzu trat vor geraumer Zeit eine von außen an den Raum herangetragene Handlungsoption insofern, als über bürgerschaftliche Initiativen die Bildung eines „Westharz"-Kreises in die Diskussion eingeführt wurde, zumal einige Gemeinden im Kreis Osterode ohnehin den Anschluss an den Kreis Goslar suchen. Angesichts laufender Gespräche und diverser, sich in Teilen widersprechender Initiativen wird davon abgesehen, die einzelnen vorgetragenen Optionen hier näher zu bewerten. Allerdings ist darauf hinzuweisen, dass mit der Bildung eines Großkreises „Südniedersachsen“ ein Gebilde entstünde, das die kreislichen GröBenordnungen Niedersachsens sprengte und auch beträchtlich oberhalb jener Zielgröße läge, die einwohnerseitig vom Innenminister des Landes als ,zwischen 100.000 und 300.000 Einwohner" angegeben wurde. Lässt man sich auf eine „Westharz"-Diskussion ein, wäre im Übrigen deutlicher als bislang zu fragen, ob ein solches Gebilde vor allem ökonomisch lebensfähig wäre und ob die bislang eher einseitig erscheinende Ausrichtung auf den Tourismusbereich tatsächlich zukunftsfähig ist. Der Gutachter verwies in diesem Zusammenhang auch auf seine zahlreichen Versuche, Länder-Länder-Kooperationen nicht nur nicht auszuschließen, sondern sie im Gegenteil aktiv zu befördern. Dies könnte auch in diesem Fall eine ggf. wichtige Entwicklungsperspektive insofern darstellen, als ein gesamthafter „Harzkreis“, also unter Einbezug des/eines „Ostkreises“, eine zumindest mittel- wie langfristig verfolgenswerte Option böte. Allerdings ist dem sofort hinzuzufügen, dass die bisherigen Bemühungen um ein entsprechendes Zusammenwirken eher unausgeprägt sind. Während auf der einen Seite der „Nationalpark Harz“ ein gutes Beispiel dafür darstellt, dass und wie grenzüberschreitende Aktivitäten zusammengeführt werden könnten, bildet das Beispiel der Bäderentwicklung aufgrund fehlender Abstimmungsprozesse das Gegenbild. Zudem sind die Bemühungen eines Beratungsunternehmens, die Entwicklungsperspektiven für den Teilraum gesamthaft und vor allem ,nachhaltig“ zu verbessern, von bislang nur sehr überschaubaren Erfolgen gekennzeichnet.

In der Gesamtbetrachtung wird deutlich, dass das Oberzentrum Göttingen, der eindeutige „Wachstumsmotor“ der Region, nicht ohne Grund eine Überprüfung der seine Entwicklungsmöglichkeiten eindämmenden (Kreis-)Politik anregt. Will 
man mithin ernsthaft eine „Region Südniedersachsen“ in der Form eines Zusammengehens aller drei benannten Kreise, wäre zunächst die seitens der Stadt Göttingen aufgekündigte Finanzvereinbarung mit dem Kreis zügig zu erneuern und der veränderten Ausgangssituation anzupassen; ggf. empfiehlt sich hier auch ein Moderationsprozess, der allerdings materiell von dem überragenden Entwicklungspotential (und entsprechender Definitionsmacht) der Stadt auszugehen hätte. Sie „ernährt“ weite Teile des Kreises Göttingen und stellt für Südniedersachsen jenen „Wachstumsmotor“ dar, den es nicht nur zu erhalten, sondern auszubauen gilt, erst recht nach jenem Rückschlag, den der Raum über die Aberkennung des Exzellenzstatus für die Universität erfuhr.

Im Übrigen fällt auf, dass die Diskussion um die künftige Territorialstruktur Südniedersachsens im Wesentlichen von der politischen Elite im Raum getragen wird, eine darüber hinausgehende Beteiligung, sieht man von dem Engagement gelegentlicher (und nicht selten politisch gelenkter) Bürgerinitiativen ab, kaum erkennbar ist. Damit bestätigt sich ein Vorbehalt gegenüber Territorialreformen, den die Politik ernst nehmen sollte: die mangelnde bürgerschaftliche Information und Beteiligung.

\section{Großraum Braunschweig}

Mit Blick auf den Großraum Braunschweig (Stadt Braunschweig, Stadt Salzgitter, Landkreise Peine, Wolfenbüttel und ggf. auch hier Goslar) wurde zwischenzeitlich deutlich, dass die Bemühungen des Braunschweiger Oberbürgermeisters, eine der Region Hannover komplementäre Region Braunschweig zu schaffen, wohl nicht Realität werden dürften. Der Einbezug von drei kreisfreien Städten und bis zu fünf Landkreisen schüfe hier eine regionale Größenordnung, die beträchtlich über das hinaus ginge, was die relative Kleinteiligkeit Niedersachsens nahelegt und die Verflechtungsanalysen angeraten erscheinen lassen. Der Autor tendiert eher dazu, für den Großraum drei Fusionsperspektiven zu unterscheiden: zum einen die zwischen Goslar, Wolfenbüttel und Salzgitter, zum zweiten jene, die sich auf Wolfsburg, Gifhorn und Helmstedt richtet sowie drittens schließlich eine Verbindung von Braunschweig und Peine (und/oder Wolfenbüttel), wohl wissend, dass Teile des erstbenannten Landkreises eher in Richtung Hannover tendieren. Für ein Zusammengehen von Goslar, Wolfenbüttel und Salzgitter spricht die hier erneut gegebene Konstellation eines Oberzentrums und zweier entwicklungsbedürftiger Landkreise. Zudem sind die aufgezeigten Verflechtungstendenzen in diesem Teilraum vergleichsweise eindeutig und finden sich anerkennenswerte erste Ansätze zu einem gemeinsamen Vorgehen, etwa in den Bereichen Verbraucher- und Gesundheitsschutz sowie Veterinärwesen. Die hier- 
zu zunächst von Wolfenbüttel aus gemeinsam mit Goslar und der Stadt Salzgitter geplante AöR kam aufgrund eines ablehnenden Votums des Kreistages Wolfenbüttel nicht zustande; sie hätte so etwas wie einen Nukleus für das bereits im „Grundgutachten“ angesprochene Zusammengehen der drei Akteure bilden können.

\section{Wolfsburg-Gifhorn-Helmstedt}

Für die sog. „Autoregion“, also die Stadt Wolfsburg und die Landkreise Gifhorn und Helmstedt, wurde bereits im „Grundgutachten“ ein schrittweises Zusammengehen vorgeschlagen, zumal eine weitere Selbstständigkeit des Landkreises Helmstedt aufgrund der haushalterischen Probleme weder erkennbar noch vorstellbar ist. Den potenziellen Partnern wird von einem Zusammengehen durchaus vieles abverlangt, das sich im Ergebnis allerdings für alle Seiten auszahlen könnte, selbst wenn man die Aufnahme Helmstedts als Stabilisierungsleistung für einen hilfebedürftigen Dritten einschätzt, mithin Solidarleistungen der Akteure einfordert. Strittig ist die Form des Zusammenschlusses, wobei von der Stadt Wolfsburg die Eingemeindung der den Kreis Helmstedt bildenden Kommunen, von anderen eine Einkreisung der Stadt Wolfsburg präferiert wird. Beides wäre konsequenzreich, nicht nur mit Blick auf die angestrebten Entschuldungsprozesse. Die Kreislösung folgte im Übrigen der hier skizzierten Anregung, StadtUmland-Beziehungen eine zukunftsfähige Form zu geben, vor allem den konkreten Verflechtungsprozessen $\mathrm{zu}$ folgen und Optionen für weitere Gebietseinheiten offen zu halten. Letzteres richtet sich besonders auf Gifhorn, einen Kreis. der für sich allein durchaus lebensfähig ist, nach allen dem Autor vorliegenden Informationen aber einen gleichsam logischen Partner für die bislang kreisfreie Stadt Wolfsburg darstellt, was gewiss nicht nur, aber besonders deutlich in den Pendlerzahlen zum Ausdruck kommt (65.000 Einpendler täglich). Die Vorstellung, der Stadt Wolfsburg die Kreisfreiheit zu nehmen, da trotz der Bemühungen um weitere Eingemeindungen eine Einwohnerzahl von etwa 150.000 auch mittelfristig als nicht erreichbar eingeschätzt werden muss, wäre erneut mit dem Hinweis darauf zu verbinden, dass Stadt-Umland-Beziehungen den angesprochenen Veränderungen unterliegen und von einer künstlichen Scheidung der Akteure in „kreisfrei“ oder „kreisangehörig“ künftig Abstand genommen werden sollte. Gerade im Raum Wolfsburg könnte exemplarisch vorgelebt werden, wie ein Gemeindeverband zum Nutzen des Oberzentrums wie der beteiligten Kreise zu entwickeln ist, wobei der befürchtete (aber ohnehin existente) Zentralitätsschub zugunsten Wolfsburgs durch eine faire Arbeitsteilung zwischen den Beteiligten, 
unter Einschluss von Außenstellen im Fall publikumsintensiver Leistungsbereiche, selbstverständlich sein sollte.

\section{Nordostniedersachsen}

Für Nordostniedersachsen gilt, dass sich mit Blick auf die erkennbaren Entwicklungsprobleme des Raums als letztlich überzeugende Vorgehensweise doch ein Zusammengehen der Landkreise Lüneburg, Uelzen und Lüchow-Dannenberg anbietet. Wie bereits im „Grundgutachten“ angesprochen, findet sich hier mit dem Landkreis Lüneburg eine stabile Ausgangssituation, die vor allem durch die kreisangehörige Stadt Lüneburg ihre durchaus dynamische Weiterentwicklung erfährt. Die Erweiterung des Territoriums um die Kreise Uelzen und LüchowDannenberg bietet sich vor allem aufgrund der überzeugenden Verflechtungsbeziehungen zwischen den Teilräumen an, ist allerdings dadurch erschwert, dass Lüneburg mit der Aufgabe, zur Gesundung der beiden anderen Gebietskörperschaften beizutragen, zweifellos überfordert wäre. Der Autor hat deshalb eine nachhaltige strukturpolitische Sonderförderung des Gesamtraums vorgeschlagen, die sich vor allem auf den Infrastrukturausbau, eine erweiterte Länder-LänderKooperation und, nicht zuletzt, auf bildungs- und energiepolitische Initiativen unter Beteiligung des Bundes - richten sollte. Letzteres verbindet sich mit einer proaktiven Nutzung der Strukturschwächen des Raums, wobei dieser sich nach Ausrufen der „Energiewende“ in besonderer Weise für den Auf- und Ausbau regenerierbarer Industrien und darauf bezogener Ausbildungseinrichtungen anbietet. Da sich hier mit einer diesbezüglich arbeitenden Akademie bereits der Nukleus entsprechende Aktivitäten findet, empfiehlt sich eine konsequente Förderung, die den Raum zudem aus seiner Randlagigkeit befreien sollte und ihm eine begründete Entwicklungsperspektive beließe, auch über Ländergrenzen hinweg. Zwar dürfte jede Teilregion Niedersachsens für sich spezifische Bedarfe in Anspruch nehmen, doch gilt besonders für Lüchow-Dannenberg und eingeschränkter Uelzen, was andere eher punktuell erfahren: das Zusammenwirken von Randlagigkeit, infrastruktureller Unterversorgung und fehlendem Mut zum „Blick über den Tellerrand“, mithin die Kreis- und Ländergrenzen. Im Übrigen sei betont, dass der Kreis Lüchow-Dannenberg trotz der strukturell verfestigten Entwicklungsprobleme im vergangenen Jahr einen gewissen Konsolidierungsprozess einleitete, der ihn zurecht in die Nähe des Entschuldungsprogramms des Landes führte. Zwar muss bezweifelt werden, ob sich damit bereits eine nachhaltige Stabilisierung des Raums verbindet, doch erscheint es angezeigt, den zwischenzeitlichen Bemühungen die Anerkennung nicht zu versagen. 
Zur Vervollständigung des Blicks auf Nordostniedersachsen sei schließlich angefügt, dass sich eine vor allem von den Vertretern Lüneburgs präferierte Variante durchaus dann anbietet, wenn sich eine Sonderförderung des Gesamtraums nicht verwirklichen lassen sollte: In diesem Fall wäre zu erwägen, den Landkreis Uelzen zu teilen, wobei die Nordhälfte - ohnehin erkennbaren Orientierungen und Verflechtungen folgend - Lüneburg zugeschlagen werden sollte, die Südhälfte (unter Einschluss der Stadt Uelzen) dagegen dem Landkreis Celle. Die faktischen Verflechtungsprozesse lassen eine solche Variante durchaus verfolgenswert erscheinen, doch stehen dem voraussichtlich der bürgerschaftliche Wille und die parteipolitischen Mehrheitsverhältnisse entgegen. Die dritte mögliche Variante für diesen Teilraum besteht im Übrigen in der vor allem seitens des Lüneburger Oberbürgermeisters präferierten Kreisfreiheit der Stadt. Hier dominiert die Vorstellung, das sich produktiv entwickelnde Oberzentrum weiterhin auf die Metropolregion Hamburg und die hier erkennbare Entwicklungsdynamik ausrichten zu lassen, eine nicht nur verständliche, sondern durchaus auch nachvollziehbare Position, die allerdings am fehlenden Willen einiger Umlandgemeinden zur Eingemeindung und an der mit einer etwaigen Kreisfreiheit zweifellos verbundenen Bedeutungsminderung des Landkreises Lüneburg scheitern dürfte. Sollte die neue Landesregierung allerdings eine Aufwertung der städtischen Räume vorsehen, böte sich auch für Lüneburg ggf. eine der Diskussion Göttingens vergleichbare Argumentation an.

\section{Unterelbe}

Im weiteren Verlauf der Unterelbe folgen dann die Kreise Harburg und Stade, die je für sich handlungs- und zukunftsfähig erscheinen und von ihrer Hamburgnahen Lage profitieren. Gleichwohl gibt der Autor zu erwägen, bei einer „Maßstabsvergrößerung" der niedersächsischen Kreisebene die erfreuliche Ausgangssituation beider Kreise ggf. noch dadurch zu verstärken, dass bei erweiterter Gemeinschaftsarbeit und einem potenziellen späteren Zusammengehen ein höchst erfolgversprechender „Kragenkreis“ um die Freie und Hansestadt Hamburg entstünde, der die Verfolgung nicht nur der regionalen, sondern auch der niedersächsischen Landesinteressen noch wesentlich stärker zum Ausdruck bringen könnte, als dies bereits heute der Fall ist. Im Ergebnis entstünde allerdings ein Kreisgebilde, das eine Einwohnerzahl von 400.000 beträchtlich überschritte, eine wie im Fall Göttingens deutlich überprüfungsbedürftige Größenordnung. Andererseits sollte der Entwicklungsdynamik des Großraums Hamburg mit einer verbesserten Kommunikations- und Kooperationsstruktur Niedersachsens begegnet werden, was untypische auch territoriale Bezüge erforderlich machte, 
unter Einschluss der wohl selbstverständlichen Mitgliedschaft der Stadt Lüneburg, als dem einzigen niedersächsischen Oberzentrum in der Metropolregion. Vergleicht man die sich auf Hamburg richtenden Aktivitäten SchleswigHolsteins und Niedersachsens, so ergibt sich ein deutlicher Nachholbedarf für die niedersächsische Seite.

\section{Unterweser}

Der Landkreis Cuxhaven befindet sich in einer insofern komfortablen Situation, als er seine Entwicklungsperspektiven gleich zweiseitig ausrichten könnte: zum einen in Richtung der Metropolregion Hamburg, zum anderen in Richtung der Region Bremen-Oldenburg. Angesichts der soeben diskutierten Entwicklungsmöglichkeiten für Harburg und Stade sieht der Gutachter aufgrund der vorliegenden Daten das Entwicklungspotenzial Cuxhavens allerdings eher in einer Ausrichtung auf die Unterweser, also etwa in einer erweiterten Kooperation mit dem Kreis Osterholz, wobei als (allerdings externes) Oberzentrum Bremerhaven fungieren würde. Zwar verbinden sich mit einer solchen Kooperationsperspektive die seit langem bekannten Vorbehalte, doch könnte dem durch die Kreisgrenzen überschreitende Zusammenarbeitsformen, etwa den Arbeitskreis Wirtschaftsstruktur Region Bremerhaven, entgegengewirkt werden. Das Regionalforum Bremerhaven fungierte in diesem Fall als gleichsam gebietskörperschaftliches Korrelat.

\section{Metropolregion Bremen-Oldenburg}

Der erweiterte Blick auf die Metropolregion Bremen-Oldenburg macht die Schwierigkeiten einer funktionalen Abgrenzung in diesem Raum und der darüber liegenden Küstenregion deutlich. Während sich mit Blick auf den Kreis und die Stadt Oldenburg eine erweiterte Kooperation mit dem Ammerland anbietet (und von allen signifikanten Indikatoren bestätigt wird), gilt dies so nicht für die Küstenregion. Von der topographischen Lage her böte sich angesichts der erwarteten und schrittweise (hoffentlich) Realität werdenden Entwicklung des JadeWeserPorts eine die Jadebay gesamthaft wahrnehmende Kreiskonstruktion an, in die der Kreis Friesland, die Stadt Wilhelmshaven und der Kreis Wesermarsch aufgenommen werden sollten. Gleichwohl stellen sich hier Probleme, die weniger mit ökonomischen und sozialen Grunddaten zu tun haben, als vielmehr mit historisch erkennbaren Animositäten. Während die Stadt Wilhelmshaven und Friesland ihre Kooperation inzwischen nicht nur erfolgversprechend erweiterten und in eine Fusionsdiskussion eingetreten sind (für die man sich über die Vergabe eines Gutachtens eine erweiterte Legitimation verspricht), richtet sich der Kreis We- 
sermarsch eher in Richtung Oldenburg aus, ohne hier auf etablierte Kooperationsstrukturen verweisen zu können. Mit Blick auf die Stadt Wilhelmshaven ist im Übrigen zu bedenken, dass sie schon von ihrer schieren Größenordnung her den Status der Kreisfreiheit kaum mehr legitimieren kann und die erwähnten Zusammenarbeitsformen und vor allem Verflechtungsprozesse mit dem Kreis Friesland eine Aufgabe der Kreisfreiheit nahelegen. Von/an ihr würden die betroffenen Parteien gesamthaft profitieren und könnte - bei Überwindung der Animositäten - auch der Kreis Wesermarsch partizipieren. Eine Kragenkreislösung um die Jadebay böte sich in besonderer Weise an, will man die sich bietenden vor allem ökonomischen Entwicklungsmöglichkeiten vollständig ausschöpfen. Alternativ oder besser ergänzend wäre auch daran zu denken, Friesland, Wilhelmshaven und Wittmund zusammenzuführen. Dies wiederum beinhaltete einen von der Einwohnerzahl her respektablen Kreis, der zudem geeignet scheint, die erkennbare Strukturschwäche des Raums abzubauen und vor allem auch dem Kreis Wittmund eine Handlungs- und Zukunftsperspektive zu geben; das dem landsmannschaftliche Differenzen entgegen stehen, ist durchaus bekannt.

\section{Ostfriesisches Kernland}

In Richtung der holländischen Grenze wäre zu erwägen, auch hier eine eher gesamthafte Lösung zu erörtern, die die Kreise Aurich und Leer mit der Stadt Emden zu einem „Ostfriesischen Kernland“ zusammenwachsen lässt. Emden mit inzwischen kaum mehr 50.000 Einwohnern muss aufgrund akuter Finanzierungsprobleme und wenig erfreulicher demographischer Prognosen seine Kreisfreiheit überdenken, verbliebe freilich als das Oberzentrum der Region deren Wachstumspol. Zwar weist der Oberbürgermeister der Stadt kontinuierlich auf sich verbessernde ökonomische Grunddaten hin, doch ist diese verständlicherweise positive Eigensicht nicht ohne Rück- und Fehlschläge. Mittelfristig steht nicht zu erwarten, dass Emden seine Kreisfreiheit durchzuhalten vermag, auch und gerade im Vergleich zu den anderen Städten des Landes. Zudem wäre positiv zu bedenken, dass Emden als das überragende Oberzentrum der entscheidende Akteur bliebe. Erneut erweist sich angesichts gegebener Verflechtungen und sich bietender Entwicklungsmöglichkeiten die Differenzierung zwischen „kreisfrei“" und „kreisangehörig“" als wenig zukunftsorientiert.

\section{Emsland-Grafschaft Bentheim}

Vergleichsweise einfach stellt sich die Aufgabe einer Überprüfung der niedersächsischen Territorialstrukturen im Grenzraum zu den Niederlanden dar. Hier 
ergibt sich mit Blick auf den Landkreis Emsland, einen der erfolgreichsten und stabilsten Kreise des Landes, lediglich die Frage, ob sich nicht ein konsequentes Zusammengehen mit der Grafschaft Bentheim empfiehlt, zumindest in längerfristiger Perspektive. Dieser Landkreis bewegt sich auf einer gerade noch akzeptablen Größenordnung (was die Einwohnerzahl anlangt) und kann vor allem vorweisen, dass er geradezu exemplarisch eine erweiterte Länder-LänderKooperation pflegt, in diesem Fall mit den angrenzenden Niederlanden. Mittelund langfristig dürfte ein Aufgehen der Grafschaft in einem entsprechend vergrößerten Kreis Emsland wohl selbstverständlich werden, prioritär stellt sich dies allerdings nicht.

\section{Cloppenburg-Vechta}

Bei einer Rückkehr in das Zentrum des Landes bietet sich längerfristig ein Zusammengehen der ohnehin eng kooperierenden Landkreise Cloppenburg und Vechta an. Beide können je für sich als „stabil“ gelten, würden aber bei einem Zusammengehen mit knapp 300.000 Einwohnern und einer für die ländlich strukturierte Region erfolgversprechenden ökonomischen Ausgangssituation ein bemerkenswertes Zentrum bilden - auch ohne ein dominierendes Oberzentrum. Hier böte sich zudem die Möglichkeit, aufgrund der gegebenen Vorarbeiten interkommunale Zusammenarbeit zu einem Modell weiterzuentwickeln, das wesentlich positivere Wirkungen zeigt, als dies in anderen Räumen Niedersachsens bislang der Fall ist. Allerdings sei auch in diesem Kontext darauf verwiesen, dass man auf der benannten Trias Kommunikation $\rightarrow$ Kooperation $\rightarrow$ Koordination schrittweise eine wachsende Verbindlichkeit von Kooperationen anstreben sollte, die wiederum in großflächigere Arbeitsteilungen der öffentlichen Einrichtungen mündete.

\section{Stadt und Landkreis Osnabrück}

Die Stadt und der Landkreis Osnabrück schließlich beenden diesen „Rundgang“ an den Grenzen des Landes. Hier findet sich gleichfalls eine kaum zu verändernde Ausgangssituation, zumal sowohl die Größenordnungen als auch die Leistungsfähigkeit von Stadt wie Landkreis eine solide Eigenständigkeit in mittelfristiger Perspektive erkennen lassen. Das ohnehin gegebene kooperative Miteinander könnte sich auch für andere Landesteile und für das Verhältnis von Oberzentren zu ihrem Umland als durchaus exemplarisch erweisen.

\section{Diepholz und Nienburg}

Bei Verfolgung des hier vorgedachten Modells ist in der Landesmitte südlich Bremens zudem auf eine sich anbietende Kooperation der Landkreise Diepholz 
und Nienburg zu verweisen, wobei Nienburg als der wesentlich kleinere Kreis in einer ungleich schwächeren Verhandlungsposition sein dürfte und trotz der Nähe zu Hannover den Kreissitz an Diepholz verlieren könnte. Auch hier gilt, dass beide Kreise ohne ein Oberzentrum auskommen (müssen), sich gerade deshalb aber ihr Zusammengehen anbietet.

\section{Verden und Rotenburg, Heidekreis und Celle}

Dies setzt sich mit Blick auf Verden und Rotenburg fort, beides Kreise, die von der Größenordnung her ,,am Rande“ liegen und durch eine erweiterte Kooperation nur gewinnen können, ergänzt um eine Kooperation des Heidekreises mit dem Landkreis Celle. Zwar können beide Kreise eine gewisse Stabilität für sich in Anspruch nehmen, doch empfiehlt sich ein Zusammengehen nicht nur aus regionalen, sondern auch aus landesspezifischen Gründen. So ist für den Heidekreis die Nutzung Celles zur „Anbindung“ an den Großraum Hannover sicher kein Nachteil und könnte umgekehrt Celle von den Ausgleichs- und Ergänzungsfunktionen, auch in touristischer Hinsicht, des Heidekreises profitieren. Eine zweite Option wurde bereits angesprochen, letztlich aber verworfen: die Teilung des Kreises Uelzen. In diesem Fall würde der Südteil des Kreises samt der Stadt Uelzen mit dem Landkreis Celle verschmelzen, dann freilich den Heidekreis gleichsam ,partnerlos“" lassen.

\section{Weserbergland}

Dieser Überblick wird abgeschlossen mit Blick auf das Weserbergland, für das sich ein Zusammengehen von Hameln-Pyrmont, Holzminden und Schaumburg aus nahezu jeder Perspektive anbietet. Zwar weiß der Autor um die historischen Grenzen, die sich insbesondere Schaumburg in einer solchen Konstellation stellen, doch könnte das Weserbergland nur in dieser „Dreiheit“" gesamthaft entwickelt werden, nicht nur in seinen ökonomischen und sozialen Konstellationen, sondern auch und gerade mit Blick auf etwaige touristische Aktivitäten.

\section{Hildesheim und Peine}

Im Ergebnis bliebe als nahezu einziger Solitär der Landkreis Hildesheim, der aufgrund seiner Ausgangsdaten dies auch durchaus zu rechtfertigen vermag. Allerdings ist nicht auszuschließen, dass hier eine erweiterte Kooperation und ggf. ein späteres Zusammengehen mit dem Landkreis Peine angedacht werden sollte, insbesondere dann, wenn man Braunschweig als stabilen Solitär oder sich anderweitig verbindendes Oberzentrum beließe. 


\section{Delmenhorst}

Unter diesen Oberzentren verbleibt schließlich die Stadt Delmenhorst, ein aufgrund der ungeklärten Konstellation im Großraum Bremen-Oldenburg noch nicht „platzierter“ Solitär. Da sich Delmenhorst mit einer den Status einer kreisfreien Stadt kaum mehr zu rechtfertigenden Einwohnerzahl aber ohnehin in ein größeres kreisförmiges Gebilde einordnen sollte, böte sich hierfür ggf. die Mitwirkung in einem durch die Stadt und den Kreis Oldenburg sowie den Kreis Ammerland gebildeten Großkreis.

Alle der hier vorgetragenen Handlungsoptionen finden sich in dem Anfang dieses Jahres der Landesregierung vorgelegten Abschlußbericht im Detail, mithin auch und gerade im Einzelfall, begründet. Der interessierte Leser sei vor allem auf die ungewöhnlich tiefgehenden empirisch-analytischen Zugänge verwiesen, die „Abwehrhaltungen“ natürlich nicht ausschließen, sie aber erschweren sollten, zumal die Ausführungen auf einer jederzeit überprüfbaren und belastbaren Datenbasis aufbauen.

\section{b. Mögliche Vorgehensweisen}

Abschließend sei erneut darauf verwiesen, dass die vorstehend benannten Entwicklungsoptionen sich aus der Logik des jeweiligen Teilraums ergebende Handlungsmöglichkeiten darstellen, die von unterschiedlicher Bedeutung und politischer Priorität sein dürften. Insofern bietet es sich an, auf der Basis dieser zweiten (und letzten) Fortschreibung des „Grundgutachtens“ eine gewisse Priorisierung der sich bietenden Handlungsoptionen vorzunehmen, mithin besonders dringliche Fälle zu identifizieren, die im Zentrum des vom Land vorzusehenden Reformansatzes stehen könnten, vielleicht sogar sollten. Die nachfolgenden Kartierungen eines entsprechenden Vorgehens fassen die diesbezüglichen Vorstellungen des Autors zusammen, wobei zwischen einer Minimallösung, einer Entwicklungsvariante und einem Zukunftsmodell unterschieden wird; im letztgenannten Fall wird die Mittel- um eine Langfristperspektive ergänzt.

Die Minimallösung nimmt vor allem auf, was in den teilregionalen Ergänzungsuntersuchungen für Südniedersachsen, den Raum Wolfsburg-Gifhorn-Helmstedt und Nordostniedersachsen, mithin die sog. Problemregionen des Landes, zutage gefördert wurde, ergänzt um jene seit längerem erkennbaren Diskussionen im Weserbergland (Holzminden-Hameln-Pyrmont) und im Küstenraum (FrieslandStadt Wilhelmshaven). Damit fällt eine gewisse Vorentscheidung zugunsten 
eines kreislich ausgerichteten Modernisierungsansatzes (anstelle einer zu unpräzisen „Regions“- oder Regionalisierungsdiskussion) und wird zudem ein Übergang zu einer zeitgemäßen Lösung der das Land kontinuierlich belastenden Stadt-Umland-Probleme erkennbar. Letzteres richtet sich auf eine zeitnahe Überwindung der in Niedersachsen ungewöhnlich disparaten (Groß-) Stadtstruktur, die - neben dem nicht zu verallgemeinernden Solitär Hannover letztlich durch nur drei weitere Großstädte (Braunschweig, Osnabrück und Oldenburg), eine lediglich historisch erklärbare Sondersituation in Göttingen und fünf weitere kreisfreie Städte geprägt ist. Letztere liegen beträchtlich unterhalb der für kreisfreie Städte (und damit das Vorhalten einer vollständigen städtischen Verwaltung) zu erwartenden Einwohnerzahl; in wenigstens drei dieser Fälle (Wilhelmshaven, Delmenhorst und Emden) bieten sich Einkreisungen an, während für Göttingen eine Auskreisung für den Fall vorzusehen wäre, dass eine Abbildung 3: Kommunalstrukturen in Niedersachsen - Minimallösung

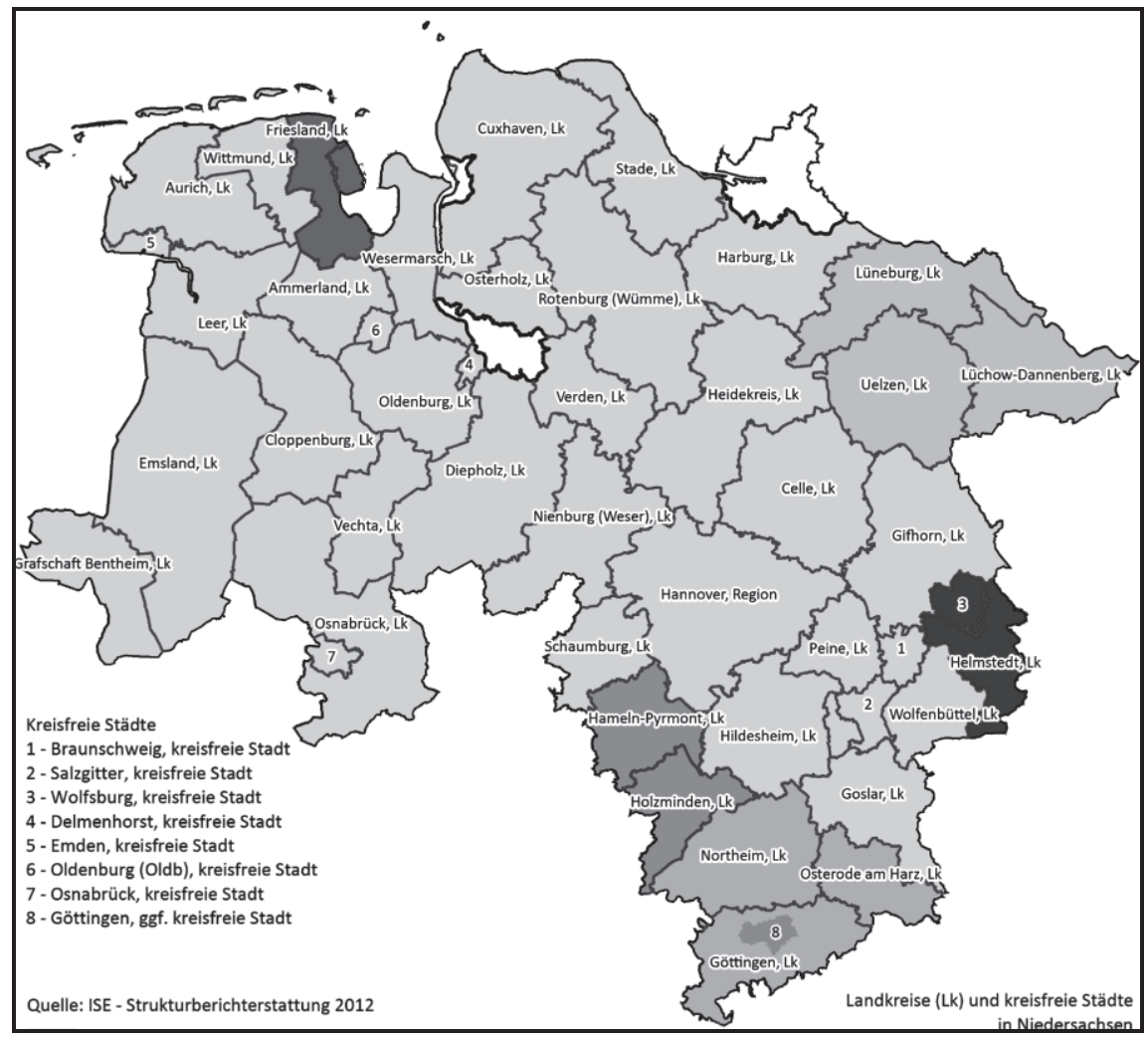


langfristig auskömmliche Finanzausstattung im Rahmen des Landkreises Göttingen nicht zu erreichen ist. Aber auch im Falle Wolfsburgs und Salzgitters empfiehlt der Gutachter ein Überdenken der Kreisfreiheit zugunsten einer die Obezentren durchaus stärkenden Nutzung der in einem größeren Gemeindeverband gegebenen Handlungsmöglichkeiten.

Die Entwicklungsvariante folgt dem und erweitert die „Minimallösung“ um eine Größerräumigkeit auch im Harz, im Großraum Braunschweig, an der Unterweser, in der Metropolregion Bremen-Oldenburg und im Küstenraum. Die vorgeschlagenen Fusionen nehmen ohnehin deutliche Verflechtungsprozesse zwischen den Akteuren auf, scheinen geeignet, das vor allem ökonomische Entwicklungspotential in den angesprochenen Teilräumen des Landes zu „optimieren“ und

Abbildung 4: Kommunalstrukturen in Niedersachsen - Entwicklungsvariante

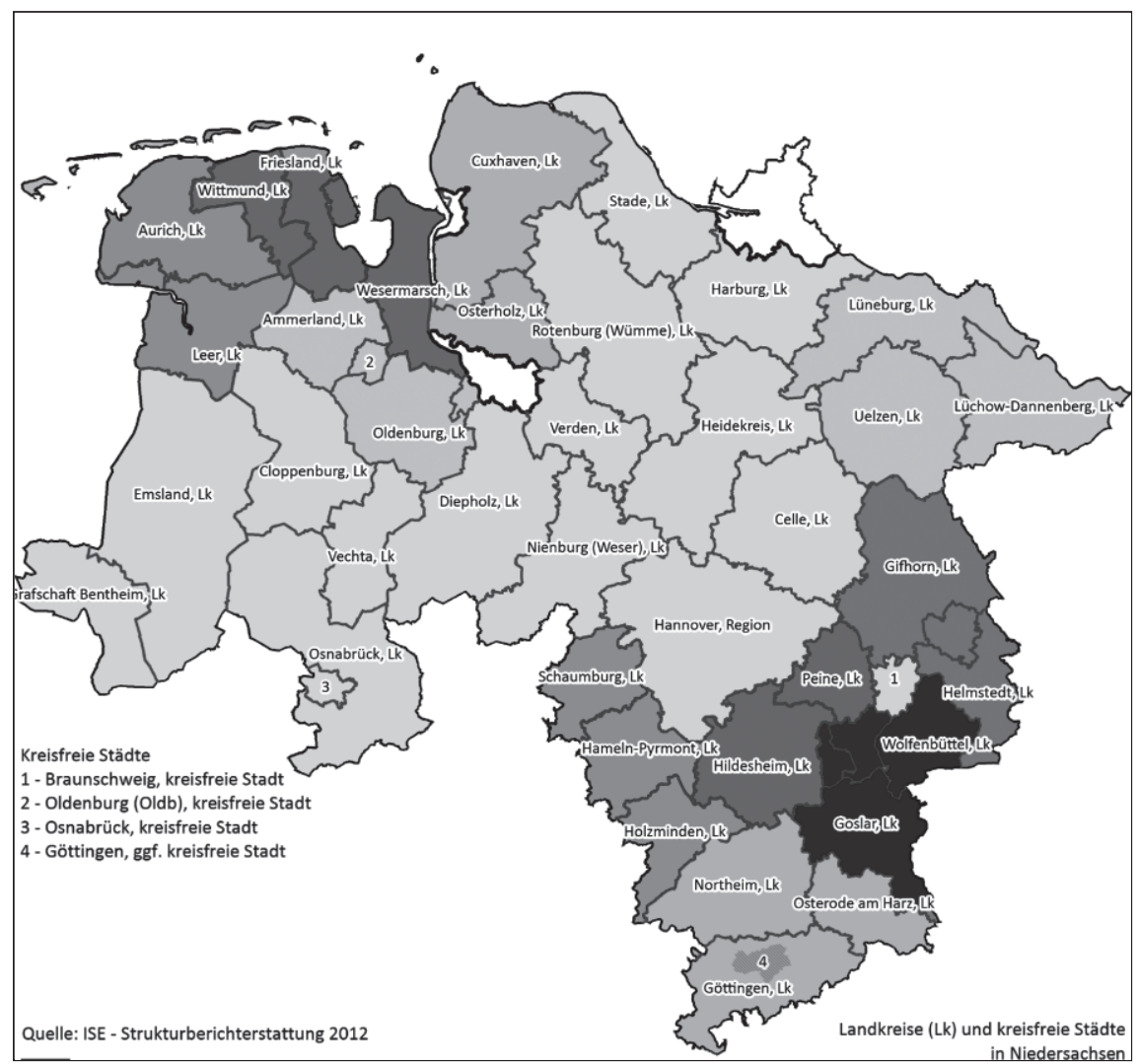


sichern angesichts der noch überschaubaren Größenordnungen durchaus eine den Namen verdienende Selbstverwaltung. Einzelvorschläge werden dabei immer strittig bleiben, so die Zuordnungen etwa Wittmunds, Schaumburgs oder Gifhorns, doch erweist sich das Fehlen überzeugender Alternativen in solchen Fällen meist als entscheidend.

Das Zukunftsmodell schließlich überträgt die Ergebnisse dieser Untersuchung auf das gesamte Landesterritorium und kommt zu einem Ausweis von insgesamt 18 gemeindeverbandlich organisierten Selbstverwaltungseinheiten, deren kreisliche Organisation sich anbietet. In den nun einbezogenen Fällen besteht meist kein dringender, aber ein sich doch anbietender Handlungsbedarf, nimmt man das politisch immer wieder betonte Bemühen um die (lokale, regionale und nationale) Wettbewerbsfähigkeit der niedersächsischen Städte, Kreise und Gemeinden

Abbildung 5: Kommunalstrukturen in Niedersachsen - Zukunftsmodell

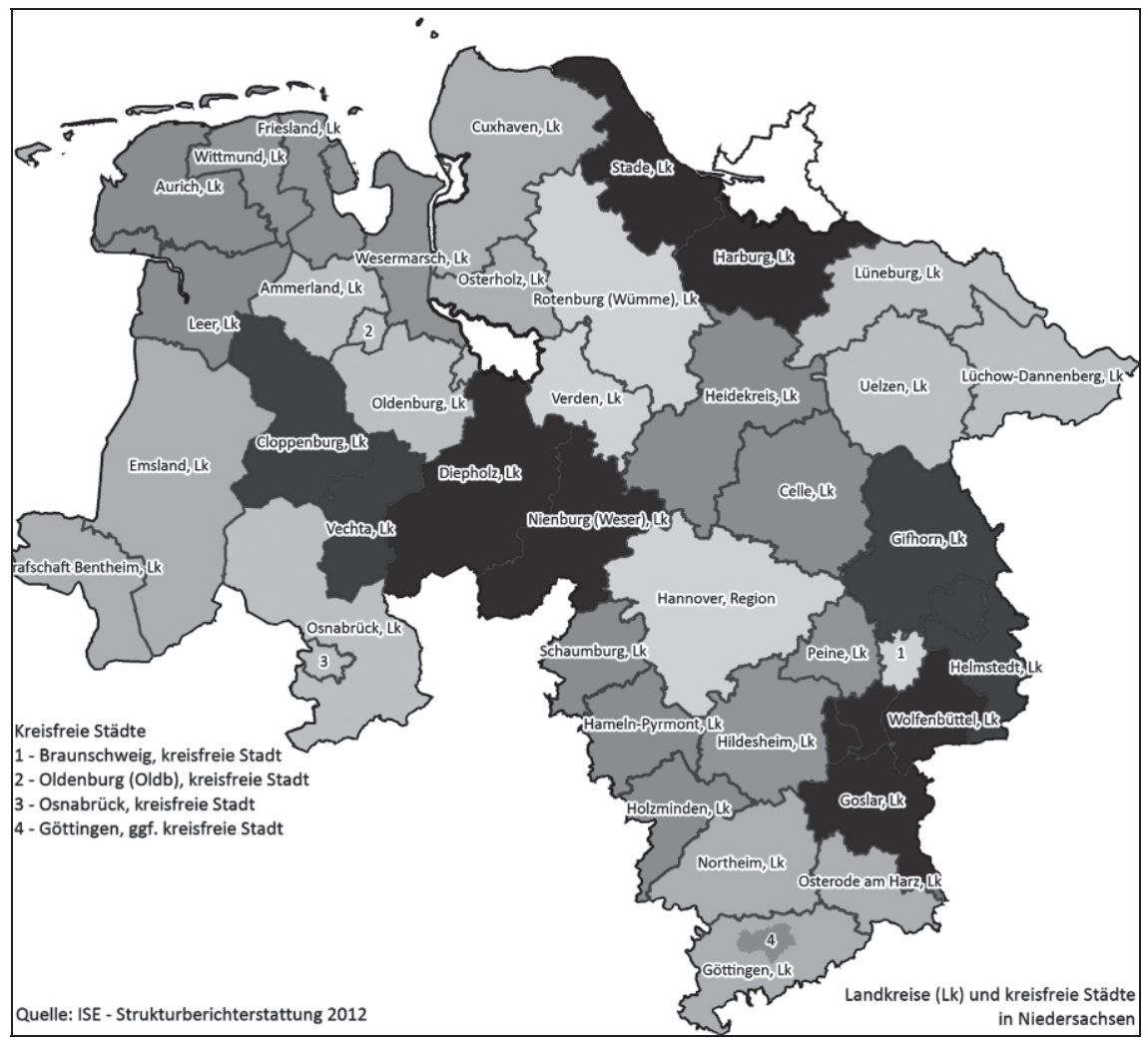


ernst. Der Gebrauch des Zukunftsbegriffs sollte allerdings nicht als „Freibrief“ für zwischenzeitliches Nicht-Handeln verstanden werden. Gerade die starken städtischen und kreislichen Akteure im Land haben bereits frühzeitig das Instrument der interkommunalen Zusammenarbeit genutzt, um die eigenen wie die regionalen Potentiale zu stärken. Dies bis hin zu auch operativ überzeugenden Arbeitsteilungen zu betreiben, erscheint unverändert angezeigt.

Ergänzt man schließlich die sich mit den vorliegenden Untersuchungsergebnissen verbindenden Optionen um jene Empfehlungen, die sich auf den konsequenten Übergang zur Einheitsgemeinde und die Herausbildung arbeitsfähiger Formen der Regionalorganisation, hier vor allem der Regionalplanung, richten, ergibt sich ein Bild der niedersächsischen Kommunalstruktur, das deutliche Züge einer handlungs- und zukunftsfähigen Selbstverwaltung trägt, ohne das für sie konstitutive bürgerschaftliche Engagement, vor allem das Ehrenamt, zu beschädigen. Einen entsprechenden Reformansatz zu verwirklichen und damit den Ruf Niedersachsens als reformorientiertes (und reformfähiges) Flächenland zu bestätigen, erscheint aufgrund der hier umfassend analysierten Ausgangssituation angezeigt. Auch von daher wäre es wünschenswert, wenn sich die politischen Parteien des Landes auf ein Gespräch über unabweisbare Reformen der Kommunalstruktur einlassen würden. Dies dürfte sich vor allem deshalb anbieten, weil es mit Blick auf die hier verfolgten Themenfelder eben nicht mehr um Erkenntnisfragen, sondern um den politischen Willen geht, defizitäre Verwaltungsstrukturen abzubauen und das Land zukunftsfähig zu halten.

Die im Landtagswahlkampf hierzu erkennbaren Positionen waren nicht so weit voneinander entfernt, wie es die öffentlichen Erklärungen gelegentlich zu suggerieren suchten. Während die zu diesem Zeitpunkt amtierende Landesregierung mit Blick auf defizitäre Kommunalstrukturen eine Fortsetzung der „Freiwilligkeitsphase“ und ggf. einen „Zukunftsvertrag II“ in Aussicht stellte, im Übrigen aber unabweisbare Reformen ankündigte, deckte sich die von der Opposition vorgetragene „Regionalisierungsstrategie“ (in Teilen) mit der hier verfolgten Ausrichtung an „Regionalen Kooperationsräumen“. Soweit erkennbar, wird damit von der ursprünglich verfolgten Idee, das Land über eine kleinere Zahl „verfasster Regionen“ zu steuern, Abstand genommen, aus der Sicht des Autors zurecht, da damit, in Ergänzung funktionaler wie rechtlicher Bedenken, eine die Selbstverwaltung schädigende Zentralisierung verbunden sein dürfte. Die darüber hinaus geplante Aufwertung der Regierungsvertretungen zu „Landesbeauftragten" und deren Ressortierung in der Staatskanzlei nahm dagegen die Ergebnisse der vom Autor vorgelegten Evaluation der Regierungsvertretungen auf; 
diese der Landesregierung seit längerem vorliegende Untersuchung votierte angesichts deutlicher und anhaltender Schwächen der Ressortkoordination für einen konsequenten Ausbau der Regierungsvertretungen zu „Entwicklungsagenturen“. Fragwürdig, ja absurd wäre es allerdings, wenn dieser Reformansatz in eine Wiedererrichtung von Bezirksregierungen mündete, deren Abschaffung eine der erfolgreichsten (und historisch bedeutsamsten) Verwaltungsreformen des Landes darstellte. 